\title{
1 A core salivary microbiome shows the high prevalence of bacterial members yet
} variability across human populations

3

4 Xinwei Ruan ${ }^{1}$, Jiaqiang Luo ${ }^{1}$, Pangzhen Zhang ${ }^{1}$ and Kate Howell ${ }^{1}$ *

$5 \quad{ }^{1}$ School of Agriculture and Food, Faculty of Veterinary and Agricultural Sciences, University of

6 Melbourne, Parkville 3010, Australia

7 Xinwei Ruan: ORCID: 0000-0002-1963-3807

8 Jiaqiang Luo: ORCID: 0000-0001-6459-3309

9 Pangzhen Zhang: ORCID: 0000-0002-9794-2269

10 *Corresponding author. khowell@unimelb.edu.au; ORCID 0000-0001-6498-0472 


\section{Abstract}

\section{Background}

25 Human saliva contains diverse bacterial communities, reflecting human health status, dietary patterns

26 and contributing to variability in the sensory perception of food. Many descriptions of salivary

27 microbiome diversity compare commonalities and differences with reference to a diseased state, but

28 the composition of healthy saliva has not been described.

29 Results

30 Here, we use a meta-analysis approach to define and explore the core membership of the human

31 salivary microbial community by collecting and re-analysing raw 16S rRNA amplicon sequencing

32 data from 47 studies with 2206 saliva samples. We found 68 core bacterial taxa that were consistently

33 detected. Differences induced by various host intrinsic and behaviour factors, including gender, age,

34 geographic location, tobacco usage, and alcohol consumption, were evident. The core of the salivary

35 microbiome was verified by collecting and analysing saliva in an independent study.

\section{Conclusion}

37 These results suggest that the methods used can effectively define a core microbial community in

38 human saliva with high prevalence. The core salivary microbiome demonstrated both stability and

39 variability among populations. Geographic location was identified as the host factor with the largest

40 effect in shaping salivary microbiota. The independent analysis underlined that the impact of

41 geographic variation is likely due to diet.

\section{Background}

44 Human saliva plays an essential role in influencing the sensory perception of foods and beverages and

45 driving the purchase decisions of consumers. When food is taken into the mouth, mixing and

46 mastication allow a semi-solid bolus to be formed, and at the same time, aroma and flavour to be

47 released from the food [1]. The variation in perceived responses can be attributed to the inter-

48 individual variability in salivary composition, especially salivary microorganisms. As a complex 
ecosystem, the human oral cavity hosts thousands of bacterial taxa, accompanied by interactions with other microorganisms [2]. It is an ecological system that contains many distinct sub-niches, including saliva, dental plaques, gingival sulcus, epithelial cells on the cheek, tongue, and teeth [3]. High heterogeneity has been reported between the composition of microbial communities that colonise on different sites [4]. The saliva is recognised as a reservoir with microorganisms from all ecological niches in the human mouth with long-term stability [5]. The ensemble of microorganisms and the expressed genetic material in human saliva is known as the "salivary microbiome".

The contribution of salivary microbiome to sensory perception of foods has been described by various studies [6-8]. However, the diverse conclusions suggest that the role of the salivary microbiome may be confounded by inter-individual variance. Meanwhile, comparing results from different studies introduces significant technical and bioinformatic biases [9] especially when studies have targeted different 16S rRNA hypervariable regions for amplification [10]. Characterising the microbial communities commonly found in most human saliva regardless of the study-specific variation could help establish the connection between salivary composition and food preference. On this basis, the shifts from a common salivary microbiome by diseases or host lifestyle factors will also be more prominent. A meta-analysis can summarise the existing knowledge and identify the commonalities and differences in salivary microbiota between people from various backgrounds.

The core oral microbiome of a healthy human has been tried to be defined for more than ten years [11]. The core microbiome is described as the common group of microbes that are important for host biological function [12]. Defining the core only depends on occupancy frequency does not necessarily reflect the underlying host-microbes functional relationship. However, it provides a foundation for prioritising members adapted to the host environment [13]. A variety of studies have been devoted to discovering the changes in human salivary microbiota based on different conditions. The variability in the microbial profile of human saliva has not only been associated with oral diseases [14-16] but also various chronic diseases that do not occur in the oral cavity, such as diabetes [17] pancreatic cancer 
76 [18] and Polycystic ovary syndrome [19]. Although the shift in human salivary composition caused by

77 diseases has been studied for decades, our understanding of the impact of host intrinsic and behaviour

78 factors is still limited.

80 Many host characteristics have been shown to have an impact on the composition of the salivary

81 microorganisms, including age [20], diet [21, 22], ethnicity [23], gender [24], smoking [25], alcohol

82 use [26], circadian rhythm [27], body mass index [28], and the type of stimulation [29]. Some studies

83 have correlated the diverse microbiome with the distinct sensory responses between consumer groups

$84[30,31]$ It has also been reported that people from different countries are colonised with distinct

85 salivary bacterial communities [32]. Li et al. analysed the human oral microbiome from Africa,

86 Alaska, and Germany and reported differences between the human groups living in various climate

87 conditions [33]. However, no study to date has demonstrated a clear global pattern in salivary

88 microbial composition.

90 In this paper, we collected raw 16S rRNA sequences of human salivary microbiota from 47 publicly

91 available datasets spanning 15 different countries. These raw data were systematically re-analysed and

92 pooled together to define a core salivary microbiome. We classified all sequences into operational

93 taxonomic units (OTUs) at 97\% identity against the Human Oral Microbiome Database (HOMD) to

94 minimise the technical variation induced by comparing data from different hypervariable regions. It

95 allowed us to make a comparison between studies and reduced the redundancy in the dataset for

96 defining the "core". Using the metadata acquired with raw data, we also investigated the influences of

97 several host factors and technical factors on human salivary microbiota. Factors that showed a

98 potentially strong impact on shaping microbial communities in saliva were selected, and the taxa as

99 potential biomarkers were identified, and linked with functional predictions. Finally, saliva samples

100 were collected from independent, healthy individuals and analysed for microbial composition to

101 confirm the results found from the global dataset. These data confirmed the composition of the core

102 microbiome members, but the verification of geographic origin was not possible. Our study

103 contributes to fundamental understandings of the stable and differential salivary microbiome across 
healthy adult populations. We have identified bacteria linked to particular identities of participants and points to salivary microbiome composition being linked to diet, rather than ethnic origin.

\section{Methods}

\section{Literature search and data collection}

109 To acquire sufficient data from healthy human saliva, available public studies related to human

110 salivary microbiota were systematically reviewed. A literature search was performed using the 111 combination of relative terms in EMBASE, MEDLINE and Web of Science for the studies published

112 before November 2020 using the terms described in supplementary data (Table S1). A supplementary

113 dataset search in NCBI's Sequence Read Archive (SRA) was also performed using the search term

114 'salivary microbiome'. The included studies met the following criteria: 1) Having samples from

115 participants without any diagnosed disease state. For studies investigating the influence of certain

116 kind of disease on salivary microbiota, only samples collected from healthy controls were included in

117 further analysis; 2) Using whole human saliva collected by spitting, swab, mouth washing or oral

118 rinsing, samples exclusively extracted from any specific oral spot, like tongue surface, parotid gland,

119 supragingival plaque, were excluded; 3) Using 16 rRNA gene high-throughput sequencing and

120 sequenced with the Illumina MiSeq platform; 4) Having and sequence file with quality score and

121 associated metadata, information about geographic locations are required; 5) Having freely available

122 sequencing data; 6) Sequencing data correctly separated according to the metadata. Raw sequence

123 data acquired from the healthy individuals of selected studies were downloaded from SRA, European

124 Nucleotide Archive (ENA), using SRA Toolkit. The files were converted to the FASTQ formats if 125 necessary.

\section{16S rRNA gene sequence processing}

127 Sequence data from each selected study were processed separately using QIIME2 (version.2020.2)

128 [34]. Sequences with primers were trimmed with "q2-cutadapt" (https://github.com/qiime2/q2cutadapt) to retain the targeted hypervariable regions. The demultiplexed paired-end sequences were 
130 firstly joined by "q2-vsearch" (https://github.com/qiime2/q2-vsearch), then subjected to a quality

131 filter with a minimum quality of Q30. The remaining reads were then clustered into operational

132 taxonomic units (OTUs) at 97\% similarity against the expanded Human Oral Microbiome Database

133 (eHOMD) version 15.1 [35] by the closed-reference OTU picking command. Reads that failed to

134 match a reference sequence in the HOMD database were discarded. The chimeras and features with a

135 frequency $\leq 10$ or detected in a single sample were also removed. The resulting tables and sequences

136 from all studies were merged by QIIME2's merge and merge-seqs commands. Samples with $<2000$

137 reads were also removed. Taxonomic annotations were assigned to the representative sequences of

138 each OTU using the HOMD database. For the factor groups containing samples with unknown

139 metadata, the unknown sample was removed from the group before the downstream analyses.

\section{$140 \quad 3$. Diversity measures in $\mathbf{R}$}

141 The merged OTU table was exported into BIOM format. Further analyses were carried out in R

142 (version 4.1.0) with custom scripts as detailed below. Samples with $>2,000$ reads were retained and

143 processed with four normalisations: 1) Rarefying samples to 5,000 (Rarefaction, RAR); 2) Samples

144 were rarefied to 5,000 and converted to relative abundance (Rarefied Total-sum Scaling, RRA); 3)

145 Samples were converted to relative abundance directly (Total-sum scaling, TSS); 4) As described by

146 Romano et al. [36], zeros were added to data through the count zero multiplicative approach using

147 the cmultRepl function of the zCompostions package [37] in R (Centred Log-ratio, CLR).

149 The alpha-diversity of all samples grouped by studies were calculated in the form of Chao1, Shannon,

150 and Simpson's diversity indices. The beta-diversity was assessed at different taxonomic levels,

151 including OTU, species, genus, family, order, class, and phylum level. For the OTU level, the beta-

152 diversity of data processed with the first three normalisations were determined using the weighted

153 UniFrac distances and Bray-Curtis dissimilarities. Euclidean distances were calculated for all

154 normalisations. At the other levels, Bray-Curtis dissimilarity was used for the first three

155 normalisations, while Euclidean distances were combined with all the normalisations. Permutational

156 multivariate analysis of variance (PERMANOVA) using the adonis2 function of vegan package [38] 
with 999 permutations was conducted to investigate the statistical differences caused by different

158 factors, adjusting for study.

\section{Defining the core microbiome}

160 The core microbiome was determined based on the abundance-occupancy pattern. Two methods

161 adapted from Shade and Stopnisek [39] and Wu et al. [13] were used. For the methods adapted from

162 the study of Shade and Stopnisek [39], samples were rarefied to 5,000. Both Bray-Curtis similarity

163 and weighted Unifrac distance were used to determine the contribution in the percentage of the

164 prospective core set to the overall beta diversity. For the method adapted from $\mathrm{Wu}$ et al. [13], OTUs

165 were filtered out with the mean relative abundance (MRA) bigger than $0.1 \%$ and the presence in more

166 than $75 \%$ of samples or $100 \%$ occupancy in more than 10 studies. To investigate the bacteria-bacteria

167 interactions in salivary microbial communities, the co-occurrence network was constructed using

168 pairwise Spearman's correlation based on relative abundance. The Spearman's correlation was

169 calculated using the rcorr function in the Hmisc R package [40] and visualised by Cytoscape v3.8.2

170 [41]. A correlation with Spearman's correlation coefficient $>0.5$ or $<-0.5$ and $p$-value $<0.01$ is

171 considered as statistically robust and shown in the network.

\section{Differential abundance analyses}

\section{$173 \quad 5.1$ Random Forest}

174 The normalised abundance of taxa in the phylum, class, order, family, genus, species and OTU level

175 were classified against each provided metadata categories to determine which factor has the largest

176 effect on the salivary microbiota. A random forest classifier was created in $\mathrm{R}$ using the randomForest

177 package [42] with default parameters. We used the randomForest (importance $=$ TRUE, proximity $=$

178 TRUE) function to generate the classification model for seven categories. For four categories that

179 have the random forest classifier with average error rate lower that $20 \%$, including hypervariable

180 region (5.0\%), geographic location (10.4\%), tobacco usage (12.7\%), sample type (13.8\%),

181 differentialt taxa were defined using cross-validation. Cross-validation was performed by the $r f c v()$

182 function for selecting appropriate features. The varImpPlot function was used to show the importance 
183 of features in the classification. The importance of features and the cross-validation curve were

184 visualized by using the ggplot2 package [43] in R.

$185 \quad 5.2$ Analysis of Compositions of Microbiomes with Bias Correction (ANCOM-BC)

186 ANCOM-BC [44] were performed to identify the taxa with different relative abundance between

187 Chinese and Western samples. Function $A N C O M B C$ were used with holm-bonferroni false discovery

188 rate correction and other default parameters. The hypervariable regions used by different studies were used as the covariate.

\section{Functional prediction}

191 Microbial metagenomes were inferred from 16S rRNA gene-based bacterial profiles, and the

192 functional prediction were conducted based on Kyoto Encyclopedia of Gene and Genomes (KEGG)

193 database [45] using the default pipeline in Phylogenetic Investigation of Communities by

194 Reconstruction of Unobserved States 2 (PICRUSt2) (Douglas et al., 2020). The ANCOM-BC analysis

195 was used to identify the differential abundant KEGG pathways by geographic location, adjusting for

196 hypervariable regions. At the same time, a random forest model was established for distinguishing

197 Chinese and Western samples, and the importance of pathways was measured using mean decreased

198 accuracy. Spearman's correlation was performed to assess the relationship between the relative

199 abundance of differential pathways and genera. The significant correlations were visualised using the corrplot package [46] in R.

\section{Comparison between Chinese and Western people on an independent cohort}

202 Saliva samples were collected from 26 participants (aged 20-60 years) recruited for a wine assessment

203 experiment and consisted of 13 Chinese and 13 Western wine experts (Table 1). The study was

204 approved by the Office for Research Ethics and Integrity of the University of Melbourne (Ethics ID:

205 1852616). Each group had six female panellists and seven male panellists. The Western panellists were defined as people who have lived in Australia for more than ten years. Chinese panellists were

207 defined as people who were born in China and had lived in Australia for no more than 18 months.

208 Bacteria genomic DNA was extracted from human saliva using QIAGEN ${ }^{\circledR}$ MagAttract ${ }^{\circledR}$ PowerSoil $\AA$

209 DNA KF Kit [47] and subjected to 16S rRNA amplicon sequencing on the Illumina platform 
210 following the Earth Microbiome Project protocols (https://earthmicrobiome.org/protocols-and-

211 standards/16s/). The raw data are available in NCBI Sequence Read Archive, with accession number

212 PRJNA786805.

213

214 The raw sequences were processed using the same pipeline in the meta-analysis as described in section 2. Additionally, raw sequencing reads were denoised into zero-radios OTUs (zOTUs) by

216 UNOISE3 pipeline [48], and taxonomically classified by classifiers trained on the full-length 16S

217 rRNA gene SILVA v138 [49] database and eHOMD v15.22 [35], respectively. The affiliation

218 between each ZOTUs and the originating OTU was determined using a customised code adapted from

219 Stopnisek and Shade [50] and available at

220 https://github.com/XINWEIR/SalivaryMicrobiome_MetaAnalysis. The relative abundance of taxa at

221 the genus level in this cohort was used as the test set for the random forest model trained using the genus-level OTU assignment information in the meta-analysis.

223

\section{Results}

\section{Inclusion of studies and sequences}

226 In this study, we extracted the 16S rRNA gene amplicon sequencing data of healthy human saliva

227 from 47 studies (Figure 1A). We abstracted data from subjects who had no diagnosed disease state,

228 hereafter named "healthy". Of course, subjects could have had subclinical diseases or may have

229 altered health status for undisclosed reasons, but we considered that this would be true of the wider

230 human population and therefore able to be included in our study. A total of 107,005,868 high quality

231 16S rRNA sequences were obtained. After removing all samples below 2,000 reads, 2206 samples

232 with 909 features were retained. The retained samples included studies from 15 countries (Table S2).

233 Most studies were conducted in three geographic regions: North America, Europe, and China (Figure

234 1B). Most sequences included in this meta-analysis were generated from the hypervariable region

235 "V3-V4" and "V4". Samples from studies targeted at "V1-V2" or the "V4-V5" region were classified

236 as "others" (Figure 1C; Table S3). Similarly, saliva samples collected by unconventional methods like 
"swab" were also classified as "others". People who recorded a smoking habit, whether they smoke ecigarettes or tobacco, were categorised as smokers. Similarly, people who drink alcohol, regardless of

239 the frequency or the type of alcohol consumed, were classified as drinkers. The given age was treated as a categorical factor, classifying into " $18-30 "$, " $31-55$ ", and " $56+"$. The samples without associated

241 information to a particular category or classified as "others" were excluded for downstream analyses related to the impact of this category. For example, the following analyses measuring the effect of hypervariable region on microbial profiles included only comparisons between the V3-V4 and V4 region (Figure 1C).

\section{Intrinsic and lifestyle factors have a significant effect on the host salivary microbiome}

246 Large variability between studies was observed in the number of reads, taxonomic profile, and alpha

247 diversity (Figure 2; Figure S1). Phylum Bacteroidetes, Proteobacteria, Firmicutes, Fusobacteria were dominated among all studies, while their proportion varies (Figure 2A). When studies were grouped by the geographic locations they originated from (coloured in Figure 2B, C, D), there is generally no difference between their intra-community diversity, represented by Shannon, Chao1, and Simpson indices. Only one study conducted in Qatar showed relatively lower Chao 1 index and higher Simpson's diversity indices than studies from other locations. However, it is hard to decide whether such variation is caused by the geographic location or other technical variations.

Because of the large disparity of methodologies amongst the studies used in our global analysis, we applied several different strategies for normalisation as described in Methods. When investigating the influences of different categories using permutational multivariate analysis of variance (PERMANOVA) tests, these normalising methods were combined as appropriate with different distance metrics, including Bray-Curtis, weighted UniFrac, and Euclidean distance. Overall, the effect of rarefaction (RAR), total-sum scaling (TSS), and the rarefied relative abundance transformation (RRA) were very similar in the result of PERMANOVA (Figure 3A-D; Table S4). Meanwhile, the centred log-ratio transformation (CLR) enlarged the variance induced by an unwanted technical

263 factor, namely the amplified hypervariable region, at all taxonomic levels. 
265 The beta-diversity analyses showed that all metadata categories measured have a significant $(\mathrm{p}<0.001)$

266 effect on the bacterial profile of human saliva at all taxonomic levels, adjusted for the study effect

267 (Table S4). However, only limited variation among samples has been explained by these factors $\left(\mathrm{R}^{2}<\right.$

$26810 \%$ ). In contrast, "study" accounts for around 35\% of the variability between samples. At the OTU

269 level, the combination of weighted UniFrac distance and the total-sum scaling (TSS) transformed data

270 best minimised the variability raised by different hypervariable regions (Figure 3D). The results of

271 the unconstrained principal coordinate analysis $(\mathrm{PCoA})$ are in agreement with the results of

272 PERMANOVA. When using Bray-Curtis dissimilarity and Euclidean distance, the samples separated

273 distinctly according to the hypervariable regions in PCoA plots, whereas the plot constructed using

274 the weighted UniFrac revealed the clusters formed by the samples from different geographic locations

275 (Figure S2). A distinct separation of samples from three main geographic locations (Figure 3F), with

276 more than half (58.0\%) of the variance explained by the first two dimensions, using weighted UniFrac

277 distance. In contrast, the differences between locations were confounded by which hypervariable

278 regions were sequenced in the PCoA plot for Bray-Curtis dissimilarity (Figure 3E). The results

279 suggest that host intrinsic and lifestyle factors significantly influence the microbial profile in human

280 saliva, regardless of the variation induced by technical factors.

\section{A core microbiome is defined from saliva from healthy humans}

282 Despite the large intra- and inter-study variability, many OTUs still showed a consistently high

283 presence and relative abundance across studies (Figure S3). These persistent OTUs detected across

284 studies with different protocols could be functionally important for the salivary microbiome of healthy

285 adults. We wanted to identify the most widespread microbial taxa within a specific population that

286 allows us to better understand the broad structure of microbiomes and their potential functional

287 consequences [12]. The abundance and occurrence frequency of taxa are two important criteria used

288 to define the "core salivary microbiome". Conventionally, thresholds on these two parameters filter all

289 taxa detected, and taxa that meet both criteria can be classified as the core salivary microbiome [13].

290 Recently, a more standardised procedure based on abundance-occupancy distribution was proposed 
291 [39]. We employed both strategies to define the core salivary microbiome to identify the microbial

292 features with high persistence and robustness in human saliva. Considering the sequences involved in

293 this meta-analysis were collected from studies targeted at different hypervariable regions, close-

294 referenced clustering at 97\% identity were used to cluster sequences into OTUs. In addition, taxa

295 defined at 100\% sequence identity may increase the redundancy in the dataset [39]. Therefore, the

296 core salivary microbiome was defined using the clustered OTUs at 97\% identity. To begin, the core

297 OTUs were determined by filtering all OTUs based on mean relative abundance and occurrence

298 frequency using the criteria described in the Methods [13]. In total, $11.6 \%$ of all OTUs (105 OTUs)

299 were included as Core 1 (Figure 4A: MRA + OCC; Table S5). Meanwhile, according to the method

300 proposed by Shade and Stopnisek [39], OTUs were ranked depending on their occupancy across

301 studies, and the contribution of top-ranked OTUs to beta-diversity was expressed by Bray-Curtis

302 similarity and weighted UniFrac distance. Two groups of the core microbiome were prioritised by

303 these two indices, to give different inclusions in the core, consisting of the top 69 OTUs (using

304 weighted UniFrac; Figure 4A: BC) and 94 OTUs (using Bray-Curtis; Figure 4A: wUF) OTUs (Figure

305 S4).

306

307 Overall, sixty-eight OTUs were shared across all three methods (Figure 4A), accounting for 7.5\% of

308 all OTUs detected and $72.5 \%$ of all $16 \mathrm{~S}$ rRNA gene sequences after clustering and filtering (Figure

309 4B, C). Firmicutes account for nearly half (46.4\%) of all core OTUs, while only one OTU belongs

310 to Saccharibacteria. The mean relative abundance (MRA) of each OTU in sub-groups classified by

311 different factors was also measured (Figure 4D). On average, the core OTUs were highly prevalent

$312(73.2 \% \pm 3.4 \%$ of cumulative relative abundance) in saliva samples across different levels in

313 subgroups classified by age, gender, geographic locations, hypervariable regions, sample type,

314 smoking, and drinking habits. The core OTUs were clustered into four main groups based on their

315 distribution pattern in sub-groups (Figure 4E). The eight OTUs affiliated to Cluster 1 showed overall

316 high abundance in all sub-groups. Cluster 2 consists of core OTUs with a slightly lower mean relative

317 abundance than Cluster 1 and higher intra-group variability. Notably, although having a higher MRA

318 than some members of Cluster 1, "476_9291" was still classified as Cluster 2. The reason could be its 
biased presence in sub-groups. For example, the relative abundance of "476_9291" is higher in samples from China than other two locations. The other two clusters contain OTUs with lower MRA than Cluster 1 and 2, while variations can still be observed within sub-groups.

Bacteria-bacteria interactions play an important role in shaping microbiota. Therefore, co-occurrence network analysis could be a useful approach for finding the most important part of the microbial community. We applied a network analysis built by Spearman's correlations to investigate whether the core OTUs defined were also important to the structure of the co-occurrence pattern. A correlation with Spearman's correlation coefficient $>0.5$ or $<-0.5$ and $p$-value $<0.01$ is considered statistically robust [51,52]. The resulting co-occurrence network contains 293 nodes and 1,424 significant correlations (edges) (Figure S5). Small modules with less than seven nodes were not displayed. Although most core OTUs have relatively low connectivity, they tend to associate with each other rather than with rare OTUs. Nine OTUs were identified as potential "hub" OTUs based on their centrality and the number of links in the network (Figure S5, 6). Because of the central position in the network, the hub taxa are regarded as the key contributor to community stability. Two core OTUs were also identified as hub taxa in the network, which are "122BU057" (Megasphaera micronuciformis) and "524_3631" (Veillonella atypica). Compared to other "hub" taxa, they showed lower connectivity and relatively high betweenness centrality (Figure S6).

\section{Geographic location is the host factor with the largest impact on bacterial composition}

338 To investigate which metadata category has the largest impact on the salivary microbiome, we

339 established random forest models to link the seven categories described above and a new category,

340 study, with the salivary microbiota data at seven taxonomic levels (OTU, phylum, class, order, family,

341 genus, and species). The effect of four normalisation methods was compared using the error rate

342 generated by random forest classification. In total, 224 random forest models were constructed

343 (Figure 5A). Among four normalisation methods, total-sum scaling produced the models that were, on 344 average, the most accurate. Generally, the random forest models built with microbial communities at

345 OTU levels have the lowest error rate $($ mean $=13.0 \%)$, while the models constructed at phylum levels 
346 have the highest (mean $=26.8 \%$ ). The model built with the hypervariable region used for sequencing

347 was also the category that showed the lowest error rate (Figure 3G). Geographic locations

348 demonstrated the second important impact on the bacterial communities, with the lowest error rate

349 among biological factors. The random forest model constructed by the other two categories, sample

350 type and tobacco usage, also showed a relatively lower error rate than other categories. Study constructed the models with high error rates at phylum $(44.3 \% \pm 2.5 \%)$ and class level $(29.3 \% \pm$ taxonomic levels, reaching $10.5 \% \pm 5.1 \%$ at OTU level. Gender and age range led to poorly performing models.

We wanted to determine whether the defined core microbiome could be used as biomarkers to differentiate people categorised by intrinsic and lifestyle factors. The random forest models showed high accuracy at OTU level were used (i.e., geographic location and smoking factors). The differential

359 OTUs induced by hypervariable regions and sample types were also analysed to exclude the influence

360 of technical factors. We further performed ten-fold cross-validation five times to measure the

361 importance of OTUs used to train the model. All OTUs before the point that the cross-validation error

362 curve starts to stabilise were defined as important OTUs. In total, we defined 59, 57, 34 and 70

363 important OTUs as biomarkers to differentiate samples according to geographic location, smoking

364 habit, hypervariable region, and sample type, respectively (Figure S7). Of these, 28, 10, 13 and 22

365 biomarkers were also classified as "core" (Figure 5B; Figure S8). Although 31 core OTUs showed the

366 importance in discriminating samples according to geographic location and smoking, nearly half of

367 them (15 OTUs) had the possibility of being confounded by technical factors (Figure 5B). After

368 excluding the OTUs that could be influenced by other factors, core OTU “322AK152" (Bergeyella

369 sp.HMT_322) was the OTU with the highest contribution to the classification of samples from three

370 geographic locations. Meanwhile, "122BU057” (Megasphaera micronuciformis) showed the highest

371 importance among the core differential OTUs specific to smoking, followed by "524_3631"

372 (Veillonella atypica). We were surprised to find that these two OTUs were the only two OTUs that

373 were defined as hub taxa in the co-occurrence network analysis (Figure S6). 


\section{The salivary microbiota as biomarkers to differentiate Chinese and Western}

375 We further analysed the changes caused by geographic locations in higher taxonomic hierarchies,

376 where many differences have been revealed. Of particular interest were taxa under

377 phylum Synergistetes and Spirochaetes, Class Mollicutes and Betaproteobacteria,

378 Family Clostridiales, and genus Prevotella. Interestingly, many taxa showed a higher relative

379 abundance in the Chinese samples, both compared to North American samples and compared to

380 European samples (Figure 6A). It suggested that the variance induced by geographic locations may be

381 dominated by the differences between samples from Chinese and Western people. Therefore, we

382 combined samples from North America and Europe into a single group, "Western." Compared to the

383 Chinese grouping, the Western group has significantly lower within-sample diversity (alpha-diversity)

384 (Wilcoxon rank-sum test, $\mathrm{p}<0.001$; Figure 6B, C). Next, we examined the differences between

385 Chinese and Western in the salivary microbiota at the genus and species level (Table S6, S7). Besides

386 establishing a random forest model, we also identified differential taxa using ANCOM-BC, adjusting

387 for the hypervariable region. We found 48 genera identified as significantly different by both methods

388 (Figure 6D, Table S6). Among them, Arachnia, Filifactor, Ottowia, Neisseria, Aggregatibacter, one

389 genus from Gracilibacteria, one genus from Clostridiales, and three other genera belonging to the

390 family Peptostreptococcaceae were strongly (standardized effect size $>10$, mean decreased

391 accuracy $>10$ ) enriched in Chinese samples.

392 Meanwhile, Prevotella, Scardovia, Bergeyell, Veillonella, Oribacterium, and one genus belonging to

393 the family Erysipelotrichaceae were strongly (standardized effect size $>7$ mean decreased

394 accuracy $>10$ ) enriched in Western samples.

395

396 Finally, we performed the functional prediction-based 16S rRNA gene profiles to investigate whether

397 differences in the salivary microbiota between Chinese and Western affect its function. Two methods,

398 ANCOM-BC, and random forest model were used to identify which pathways were differential

399 between Chinese and Western. The result of ANCOM-BC indicated that 69 pathways related to

400 metabolism were differentially abundant between the two groups. The random forest classification 
model established using KEGG pathways demonstrated an error rate of $10.01 \%$ and revealed 46

402 differential pathways. Among them, thirty pathways belonging to nine upper pathways (level 2) were

403 simultaneously defined by two methods as differing in abundance between Chinese and Western

404 (Figure 6E, Table S8). A variety of pathways was in higher abundance in Chinese samples. The

405 enrichment of these pathways in Chinese samples was mainly associated with the increased

406 abundance of Neisseria and Lautropia and the depleted abundance of Prevotella, Veillonella,

407 and Atopobium. Notably, three lipid metabolism pathways enriched in Chinese samples, including

408 "Ether lipid metabolism" (ko00565), "alpha-Linolenic acid metabolism" (ko00592), and "Linoleic

409 acid metabolism" (ko00591), have the highest standardised effect size (W statistics, Table S8). The

410 enrichment of these pathways related to lipid metabolism has been positively associated with the

411 higher abundance of Neisseria in Chinese. Neisseria may have also contributed to the pathway

412 "Carotenoid biosynthesis" (ko00906). Another metabolic pathway related to the metabolism of

413 terpenoids and polyketides, "Sesquiterpenoid and triterpenoid biosynthesis" (ko00909), showed a

414 positive correlation with a genus belong to Peptostreptococcaceae. In contrast, only one pathway

415 named "Flavone and flavonol biosynthesis" (ko00944) was enriched in the saliva samples from

416 Western. A strong positive correlation has been demonstrated between this pathway and the increased

417 abundance of Veillonella in the samples from the Western grouping.

\section{6. Validation of the core in an independent Australian cohort}

419 To validate the prevalence of the core OTUs in human saliva, we collected saliva samples from 13

420 Chinese and 13 Western participants in Melbourne and sequenced the extracted DNA with 515F-

421 806R primers. In total, 841,188 high-quality 16S rRNA sequences were obtained, which clustered into

422397 OTUs with $97 \%$ identity to the HOMD database. Among them, the core OTUs we defined in the

423 meta-analysis showed high relative abundance $(78.3 \pm 6.9 \%)$ in all collected samples. To increase the

424 accuracy of the OTU assignment, we denoised the sequencing reads using the UNOISE3 pipeline and

425 generated ZOTUs with 100\% sequence identity. After re-clustering the core OTUs defined in the

426 meta-analysis to ZOTUs, we observed that 59 of the identified OTUs in this independent dataset

427 consisted of 87 ZOTUs, and thus made up close to $80 \%$ of the relative abundance (Figure 7A). 
428 Although some sequences belonging to the same ZOTU are clustered to different OTUs, all the core

429 OTUs contain at least one highly abundant ZOTU. The taxonomic profiles of the global core

430 annotated by the HOMD database and the ZOTUs annotated by the SILVA database were very

431 similar at the genus level (Figure 8A).

432

433 We wanted to verify the observed differences between the OTUs in saliva samples from Chinese and

434 Western people in this independent dataset. Although two groups did not differ significantly when

435 considering the Shannon diversity index (Wilcoxon rank-sum test, $\mathrm{p}=0.073$; Figure 8B), the Chinese

436 group showed a higher Chao 1 Index than the Western group (Wilcoxon rank-sum test, $\mathrm{p}<0.001$;

437 Figure 8C), which is in agreeance with the result of the meta-analysis. Meanwhile, no significant

438 differences were observed between the two groups by beta-diversity analyses (Bray-Curtis and

439 weighted uniFrac distance, Table S9). We used the random forest classification model constructed

440 using the genus level profile of the large-scale dataset to predict the Chinese and Western samples in

441 this independent study. This dataset's genus-level relative abundance table was prepared from both the

442 OTU table with 97\% identity to HOMD v15.1 database (Figure 8D) and the ZOTU table annotated by

443 the HOMD v15.22 database (Figure 8E). The accuracy of both predictions was relatively low, with

$44457.7 \%$ for the OTU table and 50\% for the ZOTU table. Interestingly, most Western samples were

445 correctly classified, while most samples from Chinese participants were classified as being 'Western'

446 in this analysis.

448 Discussion

449 There is ample knowledge on the disease-affected salivary microbiota, yet our perspectives to the

450 bacteria present in healthy humans remains limited. Our systematic selection of studies, together with

451 the re-analysis of the 16S rRNA amplicon sequencing data from 47 studies offers a comprehensive

452 description of the salivary microbiome presented in adults without diagnosed disease. Our study has

453 defined the core members of salivary bacterial communities across 2211 samples from 47 studies and

454 has used metadata captured in these studies to investigate the role of different intrinsic and extrinsic 
factors on the occurrence of these core. It is clear that core members differ between geographic locations of collected saliva, and our analysis shows that Chinese participants are different from

457 Western participants (encompassing European and North American studies). A prediction of the

458 pathways enriched in each collective indicates that bacterial metabolic pathways are likely to

459 influence the aroma and flavour perception of foods. These results show that despite the core

460 microbial members of saliva being common across humans, there are differences, likely due to diet.

461 We suggest that the aroma and flavour of foods and beverages are likely to be differently affected in

462 healthy humans across the globe, meaning that preference and consumption of different foods is likely

463 to be prioritised. These results have important consequences for food and beverage design,

464 composition, and dietary advice across the globe.

465

466 Based on the abundance-occupancy pattern, the definition of core microbiome highlighted the persistent and conserved microbial communities in human saliva across the globe. Here, we compared two approaches adapted from two studies (references) to defining the core. The method adapted from the study of Wu et al. [13] is a relatively conventional strategy that has been chosen by many other studies investigating different ecosystems, such as soil [53], compost [54], wastewater treatment plants [55], and human's intestinal system [56]. The thresholds were simply setting on each taxon's mean relative abundance and occupancy across all samples. For studies aimed to determine the spatial or temporal core microbiome, additional thresholds will be added on the prevalence of taxa within sub-groups. However, the thresholds used by different studies are usually arbitrary. Some studies have even adopted only abundance or occupancy alone as criteria for defining the core. Therefore, a generalised approach for defining the core microbial members from diverse datasets based on abundance-occupancy was also applied in this study [39]. Rather than over space or time, we

478 determined the occupancy of OTUs according to their detection over study. When evaluating the contribution of top-ranked OTUs in occupancy to the beta-diversity of the community, we further hypervariable regions. 
482 The resulting core members identified by these methods have a lot in common. A majority of the core

483 OTUs defined by Shade's method is also included in the cores defined by Wu's method, suggesting

484 the recently developed multi-step approach is effective in determining taxa with high prevalence. The

485 general high relative abundance of the core across different sub-groups emphasised the utility of this

486 pipeline in identifying the persistent members across diverse datasets. Most of the core salivary

487 microbiota we defined had been proposed in previous studies as prevalent bacteria in the human oral

488 cavity that persistently span across different individuals [11, 57-59]. The dominant genus of the core

489 we defined, Streptococcus, Neisseria, and Prevotella, were concluded as core human salivary

490 microbiome by a recent study based on the MG-RAST data [60]. Ten OTUs belonging to genus

491 Streptococcus were included in the "core", two of which were classified to the cluster with overall the

492 highest relative abundance across all dimensions. The prevalence of Streptococcus we observed is

493 consistent with a previous study defining the healthy core from the 454 pyrosequencing results of

494 three individuals [11]. The most abundant core OTU we found, Streptococcus

495 oralis subspecies dentisani, has been documented in previous studies as potential oral health-

496 promoting organisms and being highly abundant at various oral niches of healthy humans [61, 62].

498 We further conducted a co-occurrence network analysis to investigate the role of these core

499 microbiota in shaping the microbial community and found the co-existence between many members

500 of the core (Figure S5). The presence of the rare OTU that became the hub suggests that although

501 some taxa are not persistently detected across the community, they may still be important for the

502 overall structure of the salivary microbiome. It has been proposed that the oral microbiota of healthy

503 individuals is both homeostatic and dynamic [57]. The core microbial members consistently present in

504 human saliva identified here may explain the stability of oral microbiota to some extent.

505 Moreover, we conducted an independent study to verify the prevalence of the core defined from the

506 published studies. The high relative abundance and occurrence of the original core OTUs in this

507 independent cohort suggest that the core human salivary microbiome we defined can be applied to

508 different datasets. After re-clustering the core OTUs to ZOTUs with 100\% sequence identity, we may

509 conclude that the same members constitute the core, even if different taxonomic resolution is applied. 
511 Besides the core microbiome, there are "variable" microbiota in human microbial communities, which

512 vary among individuals because of unique lifestyle and genetic factors [63]. We performed analyses

513 for beta-diversity of samples (Figure 3) and random forest classifications (Figure 5A), demonstrating

514 several factors-both technical and physiological-significantly discriminated between sub-populations.

515 Because of the high heterogeneity between studies in their methodology, large inter-study variability

516 was the main factor that affected the observed salivary microbiota [64, 65]. As one of the main

517 technical factors that may induce the variation, the impact of chosen hypervariable regions for

518 sequencing on driving microbial community structures has been confirmed by our study. In addition,

519 the selection of different primers for the same region and DNA extraction methods may also lead to

520 technical variations [66]. However, studies' choices of primers and DNA extraction protocols are

521 more diverse than hypervariable regions (Table S2), making it difficult to group them into categories

522 as simple as for the variable regions. By adjusting the analyses by study, the variability caused by

523 study-specific technical factors other than hypervariable regions could also be covered. For future

524 studies, it is important to establish a standardised DNA extraction and sequencing protocol on a global

525 scale. During the literature search, we found that the inter-study variation may also be attributed to the

526 criteria of recruiting participants. Although all samples included in this meta-analysis were collected

527 from the control groups and population without specific diagnosed disease, the definition of 'healthy'

528 varies. For example, the use of antibiotics was not always considered as an exclusion criterion, and

529 when included, different time intervals were adopted. However, for this study, we aimed to construct

530 a microbial community that reflects the salivary microbiota of real-life consumers. Therefore, samples

531 collected from individuals without known severe systemic diseases were included.

533 Besides the influence of variables related to study design, this study has also revealed the importance

534 of various host intrinsic and lifestyle factors. We observed the change in salivary microbial composition induced by smoking habits. As a recognised risk factor to oral health, the role of smoking

536 in shaping the human oral microbiome has received increasing attention [67]. Previous studies have

537 reported the change of Megasphaera micronuciformis caused by smoking in the microbial profile of 
the human tongue surface [68] and upper gastrointestinal tract [69]. In agreement with these findings, we identified a core OTU belonging to Megasphaera micronuciformis as a biomarker for reported smoking. In addition, we found the differential abundance of an OTU belonging to Veillonella atypica

541 between smokers and non-smokers, which agrees with a study where this species already found to be

542 increased in the saliva [70]. Another strong determinant of the salivary microbiome we defined was

543 "sample type". As we found in this study, the difference between the mouthwash sample and the other

544 two collection methods is greater than the difference between the stimulated and unstimulated saliva.

545 Contrary to the result of Jo et al. [29], the OTU belonging to Neisseria flava has not been identified as

546 differential taxa for the type of saliva. The microbial composition of alcohol drinkers and non-

547 drinkers was also found to be different, while the small sample size acquired hindered our ability to

548 draw large-scale downstream conclusions. Although the significant differences we observed in

549 salivary microbiota were also attributed to the gender and age of participants, the variations they explained are relatively low compared to other factors.

552 Geographic location has been identified as the host physiological factor with the largest impact on 553 salivary microbiota (Figure 5A). Although it only explained limited variability between samples' 554 microbial profiles, the observed variations were robust to the heterogeneity induced by different 555 hypervariable regions used (Figure 3E, F). To date, little is known regarding the influence of geographic locations on the human salivary microbiome. A comparative study reported the differences in saliva microbial composition in Alaskans, Africans, and Germans [33]. To our

558 knowledge, the geographically structured microbial communities have not been observed in healthy 559 human saliva based on the large-scale dataset used in this study. Our result also demonstrated several 560 core OTUs that may differentiate saliva samples from North America, Europe, and China. It suggests 561 that the global prevalent core microbiota is not necessary to be stable across populations. Given the 562 high abundance and occupancy frequency of the core microbiome, we would expect these taxa to be effective indicators to predict the geographic background of saliva donors. 
565 Due to the sometimes large differences in culture and lifestyle between Western and non-Western

566 populations, we further grouped our data into Western and Chinese samples. The comparison between

567 Western and non-Western populations has already been applied to the human gut microbiota, whereas

568 less is known about the saliva microbiota [71]. Our study found a difference in the abundance of

569 Veillonella spp between saliva from Western and Chinese people, where Veillonella was generally

570 higher in Western samples. Such differences may influence the flavone and flavonol biosynthesis

571 pathways in the oral cavity. Our previous study revealed the Western-born and Chinese-born wine

572 experts had different responses to the astringency of wine. Since flavonol is a well-known constituent

573 of wine-related to the bitterness and astringency perception [72], we would hypothesis that the

574 enrichment of Veillonella in Western may affect their sensitivity to the phenolic compounds in wine.

575 It has also been suggested that the regular consumption of flavonoid-rich foods, such as oolong tea,

576 may increase the abundance of Veillonella spp. in human saliva [73]. These influences can potentially

577 be the bridges that link the differences between Chinese and Western groups in sensory evaluation

578 and their salivary microbiota together. Because the amplicon sequencing data cannot speak directly to

579 the functional sequences of the observed difference, shotgun metagenome sequencing will be

580 necessary to verify the exact association. Prevotella abundance has previously been reported to be

581 enriched in the gut microbiota of non-Western populations [74], which is opposed to our observation

582 in saliva. However, there is substantial species-level diversity in Prevotella (Table S7), making it

583 plausible that different species belonging to Prevotella may respond differently to the geographic

584 background [75].

586 Although the independent dataset we collected did not show differences between samples taken from

587 Chinese and Western participants, the results of random forest classification may lead to some

588 interesting hypotheses. The prediction of models revealed that most of the Chinese samples in this

589 cohort were classified as Western. The donors of these samples were wine experts, of Chinese

590 ethnicity, born in China and living in Australia for no more than 18 months. Recent studies reported

591 that immigrants from Asia experience a "Westernization" of gut microbiota induced by dietary

592 acculturation $[74,75]$. We hypothesise that such a phenomenon may also happen in salivary 
593 microbiota. It may suggest that dietary pattern is a more important determinant than ethnicity in

594 shaping the salivary microbiota of the participants, leading to variation among different geographic

595 locations that we observed in the meta-analysis.

596

\section{Conclusions}

598 In summary, we have defined a core bacterial community in saliva from healthy humans, and this core

599 demonstrated both stability and variability among populations. The prevalence of the core members of

600 the saliva microbiome has been confirmed in an independent cohort. We have revealed the influence

601 of various host factors, such as geographic locations, incidence of smoking and drinking, on the

602 salivary microbiome. We also identified microbial and functional biomarkers to differentiate the

603 Chinese and Western people, underlying the potential relationship between salivary microbiota and

604 sensory perception. Results in this work will provide foundational information to inform future

605 studies to understand the similarities and differences in saliva microbial composition, potentially

606 associating oral to aroma and flavour perception of foods.

607

608 Availability of data and materials

609 The sequencing data supporting the conclusion of the meta-analysis in this article are available in

610 publicly accessible databases (full details can be found in Table S2). The sequencing data generated

611 and/or analysed during the current study are available in the NCBI Bioproject repository,

612 PRJNA786805 (https://www.ncbi.nlm.nih.gov/bioproject/786805). Original scripts generated during

613 the current study are available in Github

614 (https://github.com/XINWEIR/SalivaryMicrobiome_MetaAnalysis). 
617 This study was funded by the Faculty of Veterinary and Agricultural Sciences at the University of

618 Melbourne. JL and XR gratefully acknowledge a Melbourne Research Scholarship administered by

619 the University of Melbourne.

620 Legends-Results

621 Figure 1. Overview of literature search procedure and metadata of included studies. a) Largescale literature searching and data filtering process, followed by the number of samples submitted to the bioinformatic analyses; b) The locations of studies, the scale of symbols that reflect the number of samples of each study; c) Distribution of metadata categories.

Figure 2. Summary of taxonomic composition and alpha diversity of included studies. A) The mean community composition of each study at the phylum level; The alpha-diversity measured by B) Shannon index; C) Chao 1 index; D) Simpson's index, the colour of boxes stands for the geographic location of the studies. The horizontal bars within boxes represent medians. The tops and bottoms of boxes represent the 75 th and 25 th percentiles, respectively.

Figure 3. The variability in human salivary microbiota have been explained by different factors. Among them, hypervariable regions and geographic locations have the largest impact. The effect of the categories on the clustering of the sample was measured using PERMANOVA at four taxonomic levels: family (A), Genus (B), species (C) and OTU level (D). The colour indicates the different combinations of normalisation (TSS, Total-sum scaling; RRA, Rarefied relative abundance; CLR, Centred log ratio) and indices (BC, Bray-Curtis; EUC, Euclidean; wUF, weighted uniFrac). Because the results of rarefication (RAR) were very close to TSS and RRA, they were not displayed in the figures. Principal coordinate analysis (PCoA) with Bray-Curtis (E) and weighted uniFrac (F)

639 showing the differences between samples from North America, Europe, and China.

640 Figure 4. The core OTUs defined by abundance-occupancy pattern. A) Venn diagram showing 
core for all methods. (MRA+OCC: The thresholds were setting on mean relative abundance and occupancy to define the core; BC: The method adapted from Shade and Stopnisek using Bray-Curtis similarity; wUF: The method adapted from Shade and Stopnisek using weighted uniFrac distance). B)

645 Pie chart showing the number of the core (pink) versus other OTUs (blue) identified in percentage. C)

646 Pie chart showing the relative abundance of the core and other OTUs across all samples. D) Relative abundance of 68 core OTUs across subgroups classified by seven categories. E) Heatmap showing the log-transformed mean relative abundance of each core OTU at each level of different categories. indicates the taxonomic information of 68 core OTUs. The coloured squares between the tree and the annotation of phylum indicate the OTUs that were defined by the Random Forest model as "important" for distinguishing between different levels in each category. The bars on the outmost ring showing the mean relative abundance of each OTU. adults. A) Taxonomic hierarchies show the relative enrichment of taxa in three geographic locations at phylum through species level. Coloured nodes indicate log2-fold increase in median abundance of the group in $\mathrm{x}$-axis (pink) or $\mathrm{y}$-axis (blue). Only taxa showed significant changes (false discovery rateadjusted Wilcoxon rank sum $\mathrm{q}<0.05$ ) are displayed. B) and $\mathbf{C}$ ) Comparison of salivary microbial alpha diversity between the Chinese and Western samples, calculated by Chao1 (B: $p<0.001$, Wilcoxon rank-sum test) and Shannon index (C: $p<0.001$, Wilcoxon rank-sum test). D) Differential abundant genera identified between saliva from Chinese and Western samples. The panel on the left indicates the standardised effect sizes (W statistic) estimated via the difference on relative abundance using ANCOM-BC (taxa enriched in Western samples have a value shifted to right, whereas taxa enriched in Chinese samples have a value shifted to left); The panel in the middle shows the relative 
abundance of selected genera; the panel on the right indicates the Mean Decrease Accuracy of the

E) Spearman's correlation coefficients were calculated between each pairwise comparison of differential genus and KEGG pathway. Only significantly correlated comparisons ( $\mathrm{p}<0.01$, FDR adjusted Spearman's rank correlation) are displayed. The only Westernenriched pathway is marked in pink.

Figure 7. An independent cohort verifies the definition of core microbiome membership microbial alpha diversity between the Chinese and Western samples, calculated by Shannon (B: $p=$ 0.073, Wilcoxon rank-sum test) and Chao1 index (C: $p<0.001$, Wilcoxon rank-sum test). D) and E) classification model constructed using the genus profiles of samples in the meta-analysis. The genus level profiles of samples processed by D) closed-reference clustering with $97 \%$ sequence identity and

E) UNOISE3 denoising with $100 \%$ sequence identity were used as the test set.

\section{Legends-Supplementary Figures}

A) Mean read number of samples from each study. The dash line indicates that all samples below this depth $($ depth $=2,000)$ have been removed. B) The rarefaction curve reflects the increase of sample's Shannon index with sequencing depth. The curve was basically stabilised at sequencing depth $=2000$.

Figure S2 The effect of different combinations between normalisations and distance matrices on 
696 Figure S3 Phylogenetic tree showing the presence and absence of top 500 OTUs with highest

697 mean relative abundance. The colour strips on the innermost ring indicates which phylum the OTUs

698 belong to. The presence of coloured circles on the 17 rings in the middle indicate that an OTU was

699 found in a specific level of a sub-group. The grey bars on the outermost layer represent how many

700 studies that an OTU presented. The scale lines are used to highlight the number of 10,20 and 40.

701

702

Figure S4 The percentage contribution of the top-ranked OTUs to the beta-diversity of the

dataset. The beta-diversity is calculated for the whole dataset and for only the top-ranked OTUs using both Bray-Curtis similarity (A) and weighted uniFrac distance (B). The percentage contribution of top-ranked OTUs is calculated by dividing the beta-diversity among top-ranked OTUs using the betadiversity of the whole dataset. The dash lines indicate the last points at which the increase on the contribution is $2 \%$. All OTUs before this point (the point itself was also included) were defined as "core".

Figure S5 Bacterial co-occurrence network verifies the role of identified core salivary nodes is proportioned to the connectivity of nodes (node degree). Core OTUs from Figure 4 are indicated as yellow, while rare OTUs are in green. The edges between nodes represent the strength of the correlation (Spearman's correlation coefficient, $\rho \geq 0.5, p<0.01$ ). The shape of "hub" OTUs are indicated as squares with the OTU name displayed.

Figure S6 The Betweenness and Closeness centrality of OTUs involved in the network analysis.

718 The "hub" OTUs were identified as OTUs with either high connectivity (node degree) or centrality

719 (betweenness (A) and closeness (B) centrality). The accession number of "hub" OTUs are indicated. 
bioRxiv preprint doi: https://doi.org/10.1101/2021.12.13.471511; this version posted December $14,2021$. The copyright holder for this preprint (which was not certified by peer review) is the author/funder, who has granted bioRxiv a license to display the preprint in perpetuity. It is made available under aCC-BY-NC 4.0 International license.

722

723

724

725

726

727

728

729

730

731

732

733

734

735

736

737

738

739

740

741

742

743

744

745

746

747

748

Figure S7 The optimal number for defining the biomarker OTUs of four categories. The contribution of the OTUs used to differentiate the levels in each category on ten-fold cross-validation.

The OTUs were ranked in the order of importance. The dash lines represent the point at which the curve starts to become overall stable.

\section{Figure S8 The top important OTUs identified by Random Forest classification model}

established by the relative abundance of human salivary microbiota. The differential OTUs defined were ranked in descending order of their importance (Mean Decrease Accuracy). The colour of bars reflects the phylum level information of OTUs. The Mean Decrease Accuracy bar of core OTUs were marked with bolded black borders.

\section{Legends-Supplementary Tables}

Table S1 The terms used for searching in databases, including Medline, EMBASE, and Web of Science.

Table S2 Studies included after large-scale literature searches that met all the inclusion criteria.

Table S3 The full metadata used in this study, including 2206 samples with unique accession numbers.

Table S4 The influence of seven factors at seven taxonomic levels on human salivary microbial communities, measured by PERMANOVA with adonis2 function (permutation $=999$ ).

PERMANOVA models were adjusted for study.

Table S5 Core OTUs of adults' saliva microbiome defined by a) method adapted from Wu et al. (2019) ("1"= yes, "0"=no), b) method adapted from Shade and Stopnisek (2019). 
749 Table S6 Genus with differential abundance between samples from Western and Chinese people

750 identified by both ANCOM-BC and Random Forest model, adjusted for hypervariable regions

751 sequenced.

752

753 Table S7 Species with differential abundance between samples from Western and Chinese people

754 identified by both ANCOM-BC and Random Forest model, adjusted for hypervariable regions

755 sequenced.

756

757 Table S8 KEGG pathways with differential abundance between samples from Western and Chinese

758 people identified by both ANCOM-BC and Random Forest model, adjusted for hypervariable regions

759 sequenced.

760

761 Table S9 The differences between Chinese and Western participants in the independent cohort,

762 measured by PERMANOVA with adonis2 function (permutation =999). The PERMANOVA model

763 was adjusted for the gender and age range of participants. 
Table 1 Studies included after large-scale literature searches that met all the inclusion criteria.

\begin{tabular}{|c|c|c|c|c|c|c|}
\hline Study & Database & Accession Number & Location & $\begin{array}{c}\text { Hypervariable } \\
\text { Region } \\
\end{array}$ & $\begin{array}{c}\text { Number of } \\
\text { Samples }\end{array}$ & Sample Type \\
\hline$[76]$ & SRA & PRJNA323410 & India & V3-V4 & 51 & Unstimulated \\
\hline$[77]$ & SRA & PRJNA577839 & Europe & V3-V4 & 37 & Stimulated \\
\hline$[78]$ & SRA & SRP125370 & India & V3-V4 & 12 & Other \\
\hline [79] & SRA & PRJNA380250 & United States & V1-V2/V4-V5 & 4 & Unstimulated \\
\hline$[80]$ & ENA & PRJEB9010 & Europe & V3-V4 & 70 & Stimulated \\
\hline$[81]$ & SRA & PRJNA438728 & India & V3-V4 & 30 & Mouthwash \\
\hline$[82]$ & SRA & PRJNA321534 & United States & V4 & 18 & Unstimulated \\
\hline [83] & SRA & PRJNA361501 & Europe & $\mathrm{V} 3-\mathrm{V} 4$ & 99 & Other \\
\hline$[84]$ & ENA & PRJEB37445 & China & V3-V4 & 436 & Stimulated \\
\hline$[57]$ & ENA & PRJEB11529 & Canada & V3-V4 & 96 & Stimulated \\
\hline$[85]$ & SRA & PRJNA609244 & Europe & $\mathrm{V} 1-\mathrm{V} 2$ & 13 & Unstimulated \\
\hline$[86]$ & SRA & PRJNA503603 & China & $\mathrm{V} 3-\mathrm{V} 4$ & 24 & Unstimulated \\
\hline$[87]$ & SRA & PRJNA495719 & China & $\mathrm{V} 3-\mathrm{V} 4$ & 22 & Unstimulated \\
\hline [88] & ENA & PRJEB37299 & Europe & V4 & 11 & Stimulated \\
\hline [89] & SRA & PRJNA386665 & China & $\mathrm{V} 4$ & 127 & Unstimulated \\
\hline$[90]$ & SRA & PRJNA578492 & China & $\mathrm{V} 3-\mathrm{V} 4$ & 14 & Unstimulated \\
\hline [91] & SRA & PRJNA326866 & Europe & $\mathrm{V} 1-\mathrm{V} 2$ & 20 & Unstimulated \\
\hline$[92]$ & SRA & PRJNA484857 & China & $\mathrm{V} 3-\mathrm{V} 4$ & 15 & Unstimulated \\
\hline [93] & ENA & PRJEB21767 & Europe & V3-V4 & 47 & Stimulated \\
\hline
\end{tabular}




\begin{tabular}{|c|c|c|c|c|c|c|}
\hline$[94]$ & SRA & PRJNA586897 & China & V3-V4 & 64 & Unstimulated \\
\hline$[95]$ & SRA & SRP113577 & China & V4-V5 & 71 & Unstimulated \\
\hline$[96]$ & SRA & PRJNA292800 & United States & V4 & 28 & Unstimulated \\
\hline$[97]$ & SRA & PRJNA602902 & United States & $\mathrm{V} 3-\mathrm{V} 4$ & 119 & Stimulated \\
\hline$[98]$ & SRA & PRJNA623352 & Europe & V3-V4 & 17 & Unstimulated \\
\hline [99] & SRA & PRJNA356414 & Europe & V3-V4 & 10 & Unstimulated \\
\hline [100] & SRA & PRJNA602902 & United States & V3-V4 & 40 & Stimulated \\
\hline [101] & SRA & PRJNA587625 & Qatar & V3-V4 & 73 & Unstimulated \\
\hline [102] & SRA & PRJNA612815 & Europe & $\begin{array}{c}\mathrm{V} 1-\mathrm{V} 3 / \mathrm{V} 3- \\
\mathrm{V} 4 / \mathrm{V} 4-\mathrm{V} 5 / \mathrm{V} 6- \\
\mathrm{V} 8 \\
\end{array}$ & 44 & Other \\
\hline [103] & SRA & PRJNA413706 & United States & V4 & 30 & Stimulated \\
\hline [104] & SRA & PRJNA601054 & China & V3-V4 & 27 & Unstimulated \\
\hline [105] & SRA & PRJNA321349 & United States & V3-V4 & 20 & Stimulated \\
\hline [106] & SRA & PRJNA578951 & China & V3-V4 & 22 & Unstimulated \\
\hline [107] & SRA & PRJNA539937 & United States & V3-V4 & 25 & Other \\
\hline [108] & SRA & PRJNA421234 & New Zealand & V3-V4 & 7 & Unstimulated \\
\hline [109] & Qiita & 10823 & United States & V4 & 150 & Mouthwash \\
\hline [110] & SRA & PRJNA306560 & China & V4 & 18 & Stimulated \\
\hline [110] & SRA & PRJNA414355 & China & V3-V4 & 37 & Unstimulated \\
\hline [111] & SRA & PRJNA587078/ & China & V3-V4 & 21 & Unstimulated \\
\hline [112] & SRA & PRJNA556311 & China & V3-V4 & 20 & Unstimulated \\
\hline
\end{tabular}




\begin{tabular}{ccccccc}
\hline$[113]$ & ENA & PRJEB18476 & Europe & V4 & 11 & Stimulated \\
\hline$[114]$ & SRA & PRJNA414682 & China & V3-V4 & 20 & Unstimulated \\
\hline$[115]$ & SRA & PRJNA634162 & United States & V4 & 75 & Other \\
\hline$[116]$ & SRA & PRJNA515166 & Malaysia & V3-V4 & 72 & Unstimulated \\
\hline$[117]$ & SRA & PRJNA542018 & China & V4 & 10 & Unstimulated \\
\hline$[118]$ & SRA & PRJNA586723 & China & V3-V4 & 60 & Stimulated and unstimualted \\
\hline$[119]$ & SRA & PRJNA534340 & China & V3-V4 & 120 & Stimulated and unstimualted \\
\hline$[120]$ & SRA & PRJNA598080 & Europe & V3-V4 & 12 & Unstimulated \\
\hline
\end{tabular}




\section{References}

767 1. Mosca AC, Chen J. Food-saliva interactions: Mechanisms and implications. Trends

768 Food Sci Technol. 2017;66:125-34. https://doi.org/https://doi.org/10.1016/j.tifs.2017.06.005.

769 2. Liu D, Jiang X, Zheng HJ, Xie B, Wang H, He T, et al., editors. The modularity of

770 microbial interaction network in healthy human saliva: Stability and specificity. 2017 IEEE International Conference on Bioinformatics and Biomedicine (BIBM); 2017 13-16 Nov.

7722017.

3. Acharya A, Chan Y, Kheur S, Jin LJ, Watt RM, Mattheos N. Salivary microbiome in non-oral disease: A summary of evidence and commentary. Arch Oral Biol. 2017;83:169-73. https://doi.org/https://doi.org/10.1016/j.archoralbio.2017.07.019.

4. Mark Welch JL, Rossetti BJ, Rieken CW, Dewhirst FE, Borisy GG. Biogeography of a human oral microbiome at the micron scale. Proceedings of the National Academy of Sciences. 2016;113(6):E791-E800. https://doi.org/10.1073/pnas.1522149113. 5. Shaw L, Ribeiro ALR, Levine AP, Pontikos N, Balloux F, Segal AW, et al. The Human Salivary Microbiome Is Shaped by Shared Environment Rather than Genetics: Evidence from a Large Family of Closely Related Individuals. mBio. 2017;8(5):e01237-17. https://doi.org/doi:10.1128/mBio.01237-17.

6. Muñoz-González C, Cueva C, Ángeles Pozo-Bayón M, Victoria Moreno-Arribas M. Ability of human oral microbiota to produce wine odorant aglycones from odourless grape glycosidic aroma precursors. Food Chem. 2015;187:112-9. https://doi.org/https://doi.org/10.1016/j.foodchem.2015.04.068.

787 7. Parker M, Onetto C, Hixson J, Bilogrevic E, Schueth L, Pisaniello L, et al. Factors Contributing to Interindividual Variation in Retronasal Odor Perception from Aroma Glycosides: The Role of Odorant Sensory Detection Threshold, Oral Microbiota, and Hydrolysis in Saliva. Journal of Agricultural and Food Chemistry. 2020;68(38):10299-309. https://doi.org/10.1021/acs.jafc.9b05450.

7928 8iombino P, Genovese A, Esposito S, Moio L, Cutolo PP, Chambery A, et al. Saliva from Obese Individuals Suppresses the Release of Aroma Compounds from Wine. PLoS One. 2014;9(1):e85611. https://doi.org/10.1371/journal.pone.0085611. 2018;265:9-17. https://doi.org/https://doi.org/10.1016/j.ijfoodmicro.2017.10.028.

798 10. Soriano-Lerma A, Pérez-Carrasco V, Sánchez-Marañón M, Ortiz-González M, Sánchez-Martín V, Gijón J, et al. Influence of 16S rRNA target region on the outcome of microbiome studies in soil and saliva samples. Sci Rep. 2020;10(1):13637. https://doi.org/10.1038/s41598-020-70141-8.

802 11. Zaura E, Keijser BJF, Huse SM, Crielaard W. Defining the healthy "core 803 microbiome" of oral microbial communities. BMC Microbiol. 2009;9(1):259.

804 https://doi.org/10.1186/1471-2180-9-259.

805 12. Risely A. Applying the core microbiome to understand host-microbe systems. J Anim 806 Ecol. 2020;89(7):1549-58. https://doi.org/https://doi.org/10.1111/1365-2656.13229.

807 13. Wu L, Ning D, Zhang B, Li Y, Zhang P, Shan X, et al. Global diversity and 808 biogeography of bacterial communities in wastewater treatment plants. Nat Microbiol. 2019;4(7):1183-95. https://doi.org/10.1038/s41564-019-0426-5.

810 14. Guo R, Zheng Y, Zhang L, Shi J, Li W. Salivary microbiome and periodontal status 811 of patients with periodontitis during the initial stage of orthodontic treatment. Am J Orthod

812 Dentofacial Orthop. 2021;159(5):644-52.

813 https://doi.org/https://doi.org/10.1016/j.ajodo.2019.11.026. 
814 15. Relvas M, Regueira-Iglesias A, Balsa-Castro C, Salazar F, Pacheco JJ, Cabral C, et al.

815 Relationship between dental and periodontal health status and the salivary microbiome:

816 bacterial diversity, co-occurrence networks and predictive models. Sci Rep. 2021;11(1):929.

817 https://doi.org/10.1038/s41598-020-79875-x.

818 16. Wang K, Lu W, Tu Q, Ge Y, He J, Zhou Y, et al. Preliminary analysis of salivary

819 microbiome and their potential roles in oral lichen planus. Sci Rep. 2016;6(1):22943.

820 https://doi.org/10.1038/srep22943.

821 17. Sabharwal A, Ganley K, Miecznikowski JC, Haase EM, Barnes V, Scannapieco FA.

822 The salivary microbiome of diabetic and non-diabetic adults with periodontal disease. $\mathrm{J}$

823 Periodontol. 2019;90(1):26-34. https://doi.org/https://doi.org/10.1002/JPER.18-0167.

824 18. Torres PJ, Fletcher EM, Gibbons SM, Bouvet M, Doran KS, Kelley ST.

825 Characterization of the salivary microbiome in patients with pancreatic cancer. PeerJ.

$826 \quad 2015 ; 3: \mathrm{e} 1373$.

827 19. Lindheim L, Bashir M, Münzker J, Trummer C, Zachhuber V, Pieber TR, et al. The

828 Salivary Microbiome in Polycystic Ovary Syndrome (PCOS) and Its Association with

829 Disease-Related Parameters: A Pilot Study. Front Microbiol. 2016;7(1270).

830 https://doi.org/10.3389/fmicb.2016.01270.

831 20. Nomura Y, Kakuta E, Okada A, Otsuka R, Shimada M, Tomizawa Y, et al. Oral

832 Microbiome in Four Female Centenarians. Applied Sciences. 2020;10(15):5312.

833 21. Hansen TH, Kern T, Bak EG, Kashani A, Allin KH, Nielsen T, et al. Impact of a

834 vegan diet on the human salivary microbiota. Sci Rep. 2018;8(1):5847.

835 https://doi.org/10.1038/s41598-018-24207-3.

836 22. Kato I, Vasquez A, Moyerbrailean G, Land S, Djuric Z, Sun J, et al. Nutritional

837 Correlates of Human Oral Microbiome. J Am Coll Nutr. 2017;36(2):88-98.

838 https://doi.org/10.1080/07315724.2016.1185386.

839 23. Liu K, Chen S, Huang J, Ren F, Yang T, Long D, et al. Oral Microbiota of Children Is

840 Conserved across Han, Tibetan and Hui Groups and Is Correlated with Diet and Gut

841 Microbiota. Microorganisms. 2021;9(5):1030.

842 24. Renson A, Jones HE, Beghini F, Segata N, Zolnik CP, Usyk M, et al.

843 Sociodemographic variation in the oral microbiome. Ann Epidemiol. 2019;35:73-80.e2.

844 https://doi.org/https://doi.org/10.1016/j.annepidem.2019.03.006.

845 25. Wu J, Peters BA, Dominianni C, Zhang Y, Pei Z, Yang L, et al. Cigarette smoking

846 and the oral microbiome in a large study of American adults. The ISME Journal.

847 2016;10(10):2435-46. https://doi.org/10.1038/ismej.2016.37.

848 26. Fan X, Peters BA, Jacobs EJ, Gapstur SM, Purdue MP, Freedman ND, et al. Drinking

849 alcohol is associated with variation in the human oral microbiome in a large study of

850 American adults. Microbiome. 2018;6(1):59. https://doi.org/10.1186/s40168-018-0448-x.

851 27. Takayasu L, Suda W, Takanashi K, Iioka E, Kurokawa R, Shindo C, et al. Circadian

852 oscillations of microbial and functional composition in the human salivary microbiome. DNA

853 Res. 2017;24(3):261-70. https://doi.org/10.1093/dnares/dsx001.

854 28. Wu Y, Chi X, Zhang Q, Chen F, Deng X. Characterization of the salivary microbiome 855 in people with obesity. PeerJ. 2018;6:e4458.

856 29. Jo R, Nishimoto Y, Umezawa K, Yama K, Aita Y, Ichiba Y, et al. Comparison of oral 857 microbiome profiles in stimulated and unstimulated saliva, tongue, and mouth-rinsed water.

858 Sci Rep. 2019;9(1):16124. https://doi.org/10.1038/s41598-019-52445-6.

859 30. Cattaneo C, Riso P, Laureati M, Gargari G, Pagliarini E. Exploring Associations

860 between Interindividual Differences in Taste Perception, Oral Microbiota Composition, and

861 Reported Food Intake. Nutrients. 2019;11(5):1167. 
862

863

864

865

866

867

868

869

870

871

872

873

874

875

876

877

878

879

880

881

882

883

884

885

886

887

888

889

890

891

892

893

894

895

896

897

898

899

900

901

902

903

904

905

906

907

908

909

910
31. Gardner A, So PW, Carpenter GH. Intraoral Microbial Metabolism and Association with Host Taste Perception. J Dent Res. 2020;99(6):739-45. https://doi.org/10.1177/0022034520917142.

32. Henne K, Li J, Stoneking M, Kessler O, Schilling H, Sonanini A, et al. Global analysis of saliva as a source of bacterial genes for insights into human population structure and migration studies. BMC Evol Biol. 2014;14(1):190. https://doi.org/10.1186/s12862-0140190-3.

33. Li J, Quinque D, Horz H-P, Li M, Rzhetskaya M, Raff JA, et al. Comparative analysis of the human saliva microbiome from different climate zones: Alaska, Germany, and Africa. BMC Microbiol. 2014;14(1):316. https://doi.org/10.1186/s12866-014-0316-1.

34. Bolyen E, Rideout JR, Dillon MR, Bokulich NA, Abnet CC, Al-Ghalith GA, et al. Reproducible, interactive, scalable and extensible microbiome data science using QIIME 2. Nat Biotechnol. 2019;37(8):852-7. https://doi.org/10.1038/s41587-019-0209-9.

35. Chen T, Yu W-H, Izard J, Baranova OV, Lakshmanan A, Dewhirst FE. The Human Oral Microbiome Database: a web accessible resource for investigating oral microbe taxonomic and genomic information. Database. 2010;2010.

https://doi.org/10.1093/database/baq013.

36. Romano S, Savva GM, Bedarf JR, Charles IG, Hildebrand F, Narbad A. Metaanalysis of the Parkinson's disease gut microbiome suggests alterations linked to intestinal inflammation. npj Parkinson's Disease. 2021;7(1):27. https://doi.org/10.1038/s41531-02100156-Z.

37. Palarea-Albaladejo J, Martín-Fernández JA. zCompositions — R package for multivariate imputation of left-censored data under a compositional approach. Chemometrics Intellig Lab Syst. 2015;143:85-96.

https://doi.org/https://doi.org/10.1016/j.chemolab.2015.02.019.

38. Oksanen J, Guillaume; BF, Michael; F, Roeland; K, Pierre; L, Dan; M, et al. vegan: Community Ecology Package. R package version 2.5-7.; 2020.

39. Shade A, Stopnisek N. Abundance-occupancy distributions to prioritize plant core microbiome membership. Curr Opin Microbiol. 2019;49:50-8.

https://doi.org/https://doi.org/10.1016/j.mib.2019.09.008.

40. Frank E Harrell Jr wcfCDamo. Hmisc: Harrell Miscellaneous. R package version 4.50. 2021. https://CRAN.R-project.org/package=Hmisc.

41. Shannon P, Markiel A, Ozier O, Baliga NS, Wang JT, Ramage D, et al. Cytoscape: a software environment for integrated models of biomolecular interaction networks. Genome Res. 2003;13(11):2498-504.

42. Liaw A, Wiener M. Classification and regression by randomForest. R news. 2002;2(3):18-22.

43. Wickham H. ggplot2: Elegant Graphics for Data Analysis. 2016. https://ggplot2.tidyverse.org.

44. Lin H, Peddada SD. Analysis of compositions of microbiomes with bias correction. Nature Communications. 2020;11(1):3514. https://doi.org/10.1038/s41467-020-17041-7.

45. Kanehisa M, Goto S, Sato Y, Kawashima M, Furumichi M, Tanabe M. Data, information, knowledge and principle: back to metabolism in KEGG. Nucleic Acids Res. 2013;42(D1):D199-D205. https://doi.org/10.1093/nar/gkt1076.

46. Wei T, Simko V. R package 'corrplot': Visualization of a Correlation Matrix (Version 0.90). 2021. https://github.com/taiyun/corrplot.

47. Marotz C, Amir A, Humphrey G, Gaffney J, Gogul G, Knight R. DNA extraction for streamlined metagenomics of diverse environmental samples. BioTechniques.

2017;62(6):290-3. https://doi.org/10.2144/000114559. 
911 48. Edgar RC. UNOISE2: improved error-correction for Illumina 16S and ITS amplicon

912 sequencing. bioRxiv. 2016:081257. https://doi.org/10.1101/081257.

913 49. Quast C, Pruesse E, Yilmaz P, Gerken J, Schweer T, Yarza P, et al. The SILVA

914 ribosomal RNA gene database project: improved data processing and web-based tools.

915 Nucleic Acids Res. 2013;41(Database issue):D590-D6. https://doi.org/10.1093/nar/gks1219.

916 50. Stopnisek N, Shade A. Persistent microbiome members in the common bean

917 rhizosphere: an integrated analysis of space, time, and plant genotype. The ISME Journal.

918 2021;15(9):2708-22. https://doi.org/10.1038/s41396-021-00955-5.

919 51. Domin H, Zurita-Gutiérrez YH, Scotti M, Buttlar J, Hentschel Humeida U, Fraune S.

920 Predicted Bacterial Interactions Affect in Vivo Microbial Colonization Dynamics in

921 Nematostella. Front Microbiol. 2018;9(728). https://doi.org/10.3389/fmicb.2018.00728.

922 52. Fan P, Nelson CD, Driver JD, Elzo MA, Peñagaricano F, Jeong KC. Host genetics

923 exerts lifelong effects upon hindgut microbiota and its association with bovine growth and

924 immunity. The ISME Journal. 2021;15(8):2306-21. https://doi.org/10.1038/s41396-021-

925 00925-x.

926 53. Delgado-Baquerizo M, Oliverio AM, Brewer TE, Benavent-González A, Eldridge DJ, Bardgett RD, et al. A global atlas of the dominant bacteria found in soil. Science. 2018;359(6373):320-5. https://doi.org/doi:10.1126/science.aap9516.

929 54. Wang Y, Gong J, Li J, Xin Y, Hao Z, Chen C, et al. Insights into bacterial diversity in 930 compost: Core microbiome and prevalence of potential pathogenic bacteria. Sci Total

931 Environ. 2020;718:137304. https://doi.org/https://doi.org/10.1016/j.scitotenv.2020.137304.

932 55. Giordano C, Boscaro V, Munz G, Mori G, Vannini C. Summer holidays as break-

933 point in shaping a tannery sludge microbial community around a stable core microbiota. Sci

934 Rep. 2016;6(1):30376. https://doi.org/10.1038/srep30376.

935 56. Salonen A, Salojärvi J, Lahti L, de Vos WM. The adult intestinal core microbiota is

936 determined by analysis depth and health status. Clinical Microbiology and Infection.

937

938

939 2012;18(s4):16-20. https://doi.org/https://doi.org/10.1111/j.1469-0691.2012.03855.x.

57. Hall MW, Singh N, Ng KF, Lam DK, Goldberg MB, Tenenbaum HC, et al. Interpersonal diversity and temporal dynamics of dental, tongue, and salivary microbiota in the healthy oral cavity. npj Biofilms and Microbiomes. 2017;3(1):2.

942 58. Simón-Soro Á, Tomás I, Cabrera-Rubio R, Catalan MD, Nyvad B, Mira A. Microbial

943 Geography of the Oral Cavity. J Dent Res. 2013;92(7):616-21.

944 https://doi.org/10.1177/0022034513488119.

945 59. Yao T, Han X, Guan T, Zhai C, Liu C, Liu C, et al. Exploration of the microbiome community for saliva, skin, and a mixture of both from a population living in Guangdong. Int J Legal Med. 2021;135(1):53-62. https://doi.org/10.1007/s00414-020-02329-6.

948 60. Oliveira SG, Nishiyama RR, Trigo CAC, Mattos-Guaraldi AL, Dávila AMR, Jardim

949 R, et al. Core of the saliva microbiome: an analysis of the MG-RAST data. BMC Oral Health. 950 2021;21(1):351. https://doi.org/10.1186/s12903-021-01719-5.

951 61. Conrads $\mathrm{G}$, Westenberger J, Lürkens M, Abdelbary MMH. Isolation and Bacteriocin952 Related Typing of Streptococcus dentisani. Frontiers in Cellular and Infection Microbiology. 953 2019;9(110). https://doi.org/10.3389/fcimb.2019.00110.

954 62. López-López A, Camelo-Castillo A, Ferrer MD, Simon-Soro Á, Mira A. HealthAssociated Niche Inhabitants as Oral Probiotics: The Case of Streptococcus dentisani. Front Microbiol. 2017;8(379). https://doi.org/10.3389/fmicb.2017.00379.

63. Zarco M, Vess T, Ginsburg G. The oral microbiome in health and disease and the potential impact on personalized dental medicine. Oral Dis. 2012;18(2):109-20.

https://doi.org/https://doi.org/10.1111/j.1601-0825.2011.01851.x. 
960 64. Cornejo-Granados F, Gallardo-Becerra L, Leonardo-Reza M, Ochoa-Romo JP, 961 Ochoa-Leyva A. A meta-analysis reveals the environmental and host factors shaping the structure and function of the shrimp microbiota. PeerJ. 2018;6:e5382.

964

65. Holman DB, Brunelle BW, Trachsel J, Allen HK, Bik H. Meta-analysis To Define a

Core Microbiota in the Swine Gut. mSystems. 2017;2(3):e00004-17.

966 https://doi.org/doi:10.1128/mSystems.00004-17.

66. Wright RJ, Langille MGI, Walker TR. Food or just a free ride? A meta-analysis reveals the global diversity of the Plastisphere. ISME J. 2021;15(3):789-806.

968 https://doi.org/10.1038/s41396-020-00814-9.

969 67. Roberts FA, Darveau RP. Microbial protection and virulence in periodontal tissue as a function of polymicrobial communities: symbiosis and dysbiosis. Periodontol 2000. 2015;69(1):18-27. https://doi.org/https://doi.org/10.1111/prd.12087.

972 68. Sato N, Kakuta M, Hasegawa T, Yamaguchi R, Uchino E, Kobayashi W, et al. Metagenomic analysis of bacterial species in tongue microbiome of current and never smokers. npj Biofilms and Microbiomes. 2020;6(1):11. https://doi.org/10.1038/s41522-0200121-6. between tobacco use and the upper gastrointestinal microbiome among Chinese men. Cancer Causes Control. 2015;26(4):581-8. https://doi.org/10.1007/s10552-015-0535-2.

979 70. Karabudak S, Ari O, Durmaz B, Dal T, Basyigit T, Kalcioglu MT, et al. Analysis of 980 the effect of smoking on the buccal microbiome using next-generation sequencing technology. J Med Microbiol. 2019;68(8):1148-58. https://doi.org/https://doi.org/10.1099/jmm.0.001003.

71. Prasoodanan P. K V, Sharma AK, Mahajan S, Dhakan DB, Maji A, Scaria J, et al. Western and non-western gut microbiomes reveal new roles of Prevotella in carbohydrate metabolism and mouth-gut axis. npj Biofilms and Microbiomes. 2021;7(1):77. https://doi.org/10.1038/s41522-021-00248-x.

72. García-Estévez I, Ramos-Pineda AM, Escribano-Bailón MT. Interactions between wine phenolic compounds and human saliva in astringency perception. Food Funct. 2018;9(3):1294-309.

990 73. Liu Z, Guo H, Zhang W, Ni L. Salivary Microbiota Shifts under Sustained Consumption of Oolong Tea in Healthy Adults. Nutrients. 2020;12(4):966. 74. Vangay P, Johnson AJ, Ward TL, Al-Ghalith GA, Shields-Cutler RR, Hillmann BM, et al. US Immigration Westernizes the Human Gut Microbiome. Cell. 2018;175(4):96272.e10. https://doi.org/https://doi.org/10.1016/j.cell.2018.10.029. 75. Peters BA, Yi SS, Beasley JM, Cobbs EN, Choi HS, Beggs DB, et al. US nativity and dietary acculturation impact the gut microbiome in a diverse US population. The ISME Journal. 2020;14(7):1639-50. https://doi.org/10.1038/s41396-020-0630-6.

76. Acharya A, Chan Y, Kheur S, Kheur M, Gopalakrishnan D, Watt R, et al. Salivary microbiome of an urban Indian cohort and patterns linked to subclinical inflammation. Oral diseases. 2017;23(7):926-40.

1001 77. Anderson A, Al-Ahmad A, Schlueter N, Frese C, Hellwig E, Binder N. Influence of 1002 the long-term use of oral hygiene products containing stannous ions on the salivary 1003 microbiome-a randomized controlled trial. Scientific reports. 2020;10(1):1-8.

1004 78. Bhushan B, Yadav A, Singh S, Ganju L. Diversity and functional analysis of salivary microflora of Indian Antarctic expeditionaries. Journal of oral microbiology. 2019;11(1):1581513.

1008 salivary microbiome is consistent between subjects and resistant to impacts of short-term 
hospitalization. Scientific Reports. 2017;7(1):11040. https://doi.org/10.1038/s41598-01711427-2.

80. Cameron SJS, Huws SA, Hegarty MJ, Smith DPM, Mur LAJ. The human salivary microbiome exhibits temporal stability in bacterial diversity. FEMS Microbiology Ecology. 2015;91(9). https://doi.org/10.1093/femsec/fiv091.

81. Chaudhari DS, Dhotre DP, Agarwal DM, Gaike AH, Bhalerao D, Jadhav P, et al. Gut, oral and skin microbiome of Indian patrilineal families reveal perceptible association with age. Scientific Reports. 2020;10(1):5685. https://doi.org/10.1038/s41598-020-62195-5. 82. Chen C, Hemme C, Beleno J, Shi ZJ, Ning D, Qin Y, et al. Oral microbiota of periodontal health and disease and their changes after nonsurgical periodontal therapy. The ISME Journal. 2018;12(5):1210-24. https://doi.org/10.1038/s41396-017-0037-1. 83. Collado MC, Engen PA, Bandín C, Cabrera-Rubio R, Voigt RM, Green SJ, et al. Timing of food intake impacts daily rhythms of human salivary microbiota: a randomized, crossover study. The FASEB Journal. 2018;32(4):2060-72.

84. Debelius JW, Huang T, Cai Y, Ploner A, Barrett D, Zhou X, et al. Subspecies niche specialization in the oral microbiome is associated with nasopharyngeal carcinoma risk. Msystems. 2020;5(4):e00065-20.

85. Hijazi K, Morrison RW, Mukhopadhya I, Martin B, Gemmell M, Shaw S, et al. Oral bacterial diversity is inversely correlated with mucosal inflammation. Oral Diseases. 2020;26(7):1566-75. https://doi.org/https://doi.org/10.1111/odi.13420.

86. Ji Y, Liang X, Lu H. Analysis of by high-throughput sequencing: Helicobacter pylori infection and salivary microbiome. BMC Oral Health. 2020;20(1):84. https://doi.org/10.1186/s12903-020-01070-1.

87. Jiang Q, Liu J, Chen L, Gan N, Yang D. The Oral Microbiome in the Elderly With Dental Caries and Health. Frontiers in cellular and infection microbiology. 2019;8(442). https://doi.org/10.3389/fcimb.2018.00442.

88. Kumpitsch C, Moissl-Eichinger C, Pock J, Thurnher D, Wolf A. Preliminary insights into the impact of primary radiochemotherapy on the salivary microbiome in head and neck squamous cell carcinoma. Scientific reports. 2020;10(1):1-12.

89. Lee W-H, Chen H-M, Yang S-F, Liang C, Peng C-Y, Lin F-M, et al. Bacterial alterations in salivary microbiota and their association in oral cancer. Scientific reports. 2017;7(1):1-11.

90. Lin M, Li X, Wang J, Cheng C, Zhang T, Han X, et al. Saliva microbiome changes in patients with periodontitis with and without chronic obstructive pulmonary disease. Frontiers in cellular infection microbiology. 2020;10:124.

91. Lindheim L, Bashir M, Münzker J, Trummer C, Zachhuber V, Pieber TR, et al. The salivary microbiome in polycystic ovary syndrome (pcos) and its association with diseaserelated parameters: a pilot study. Frontiers in microbiology. 2016;7:1270.

92. Liu Y, Zhang Q, Hu X, Chen F, Hua H. Characteristics of the salivary microbiota in cheilitis granulomatosa. Med Oral Patol Oral Cir Bucal. 2019;24(6):e719-e25. https://doi.org/10.4317/medoral.23041. 93. Lundmark A, Hu YO, Huss M, Johannsen G, Andersson AF, Yucel-Lindberg T. Identification of salivary microbiota and its association with host inflammatory mediators in periodontitis. Frontiers in cellular infection microbiology. 2019;9:216.

94. Menon R, Gomez A, Brandt B, Leung Y, Gopinath D, Watt R, et al. Long-term impact of oral surgery with or without amoxicillin on the oral microbiome-A prospective cohort study. Scientific reports. 2019;9(1):1-10.

95. Niu C, Dong T, Jiang W, Gao L, Yuan K, Hu X, et al. Pregnancy-Related Ecological Shifts of Salivary Microbiota and its Association with Salivary Sex Hormones. 2020. 
96. Ozga AT, Sankaranarayanan K, Tito RY, Obregon-Tito AJ, Foster MW, Tallbull G, et al. Oral microbiome diversity among Cheyenne and Arapaho individuals from Oklahoma. American journal of physical anthropology. 2016;161(2):321-7.

97. Pushalkar S, Paul B, Li Q, Yang J, Vasconcelos R, Makwana S, et al. Electronic Cigarette Aerosol Modulates the Oral Microbiome and Increases Risk of Infection. iScience. 2020;23(3):100884. https://doi.org/https://doi.org/10.1016/j.isci.2020.100884. Assessing the impact of dental and periodontal statuses on the salivary microbiome: a global oral health scale. medRxiv. 2020.

1067 99. Russo E, Bacci G, Chiellini C, Fagorzi C, Niccolai E, Taddei A, et al. Preliminary

1068 comparison of oral and intestinal human microbiota in patients with colorectal cancer: a pilot study. Frontiers in microbiology. 2018;8:2699.

1070 100. Sierra MA, Li Q, Pushalkar S, Paul B, Sandoval TA, Kamer AR, et al. The influences of bioinformatics tools and reference databases in analyzing the human oral microbial community. Genes. 2020;11(8):878.

101. Sohail MU, Elrayess MA, Al Thani AA, Al-Asmakh M, Yassine HM. Profiling the oral microbiome and plasma biochemistry of obese hyperglycemic subjects in Qatar. Microorganisms. 2019;7(12):645. 102. Soriano-Lerma A, Pérez-Carrasco V, Sánchez-Marañón M, Ortiz-González M, Sánchez-Martín V, Gijón J, et al. Influence of 16S rRNA target region on the outcome of microbiome studies in soil and saliva samples. Scientific reports. 2020;10(1):1-13. 103. Stewart CJ, Auchtung TA, Ajami NJ, Velasquez K, Smith DP, De La Garza II R, et al. Effects of tobacco smoke and electronic cigarette vapor exposure on the oral and gut microbiota in humans: a pilot study. PeerJ. 2018;6:e4693.

1082 104. Sun X, Li M, Xia L, Fang Z, Yu S, Gao J, et al. Alteration of salivary microbiome in periodontitis with or without type-2 diabetes mellitus and metformin treatment. Scientific reports. 2020;10(1):1-14. 105. Tian N, Faller L, Leffler DA, Kelly CP, Hansen J, Bosch JA, et al. Salivary gluten degradation and oral microbial profiles in healthy individuals and celiac disease patients. Applied and environmental microbiology. 2017;83(6):e03330-16.

106. Tong Y, Zheng L, Qing P, Zhao H, Li Y, Su L, et al. Oral microbiota perturbations are linked to high risk for rheumatoid arthritis. Frontiers in cellular infection microbiology. 2020;9:475.

1091 107. Urbaniak C, Lorenzi H, Thissen J, Jaing C, Crucian B, Sams C, et al. The influence of 1092 spaceflight on the astronaut salivary microbiome and the search for a microbiome biomarker 1093 for viral reactivation. Microbiome. 2020;8(1):1-14.

1094 108. Vesty A, Gear K, Biswas K, Radcliff FJ, Taylor MW, Douglas RG. Microbial and inflammatory-based salivary biomarkers of head and neck squamous cell carcinoma. Clinical and experimental dental research. 2018;4(6):255-62.

1097 https://doi.org/https://doi.org/10.1002/cre2.139.

1098 109. Vogtmann E, Chen J, Kibriya MG, Amir A, Shi J, Chen Y, et al. Comparison of oral collection methods for studies of microbiota. Cancer Epidemiology and Prevention Biomarkers. 2019;28(1):137-43.

110. Wang T, Yu L, Xu C, Pan K, Mo M, Duan M, et al. Chronic fatigue syndrome patients have alterations in their oral microbiome composition and function. PloS one. 2018;13(9):e0203503.

111. Wang Q, Rao Y, Guo X, Liu N, Liu S, Wen P, et al. Oral microbiome in patients with oesophageal squamous cell carcinoma. Scientific reports. 2019;9(1):1-9. 
1106 112. Wang X, Zhao Z, Tang N, Zhao Y, Xu J, Li L, et al. Microbial community analysis of saliva and biopsies in patients with oral lichen planus. Frontiers in microbiology.

$1108 \quad 2020 ; 11: 629$.

1109 113. Wolf A, Moissl-Eichinger C, Perras A, Koskinen K, Tomazic PV, Thurnher D. The 1110 salivary microbiome as an indicator of carcinogenesis in patients with oropharyngeal squamous cell carcinoma: A pilot study. Scientific reports. 2017;7(1):1-10.

1112 114. Xun Z, Zhang Q, Xu T, Chen N, Chen F. Dysbiosis and Ecotypes of the Salivary

1113 Microbiome Associated With Inflammatory Bowel Diseases and the Assistance in Diagnosis

1114 of Diseases Using Oral Bacterial Profiles. Frontiers in microbiology. 2018;9(1136).

1115 https://doi.org/10.3389/fmicb.2018.01136.

1116 115. Yano Y, Hua X, Wan Y, Suman S, Zhu B, Dagnall CL, et al. Comparison of Oral

1117 Microbiota Collected Using Multiple Methods and Recommendations for New

1118 Epidemiologic Studies. Msystems. 2020;5(4):e00156-20.

1119 116. Yeo L-F, Aghakhanian FF, Tan JS, Gan HM, Phipps ME. Health and saliva microbiomes of a semi-urbanized indigenous tribe in Peninsular Malaysia. F1000Research.

1122 117. Yu FY, Wang QQ, Li M, Cheng Y-H, Cheng Y-SL, Zhou Y, et al. Dysbiosis of saliva 1123 microbiome in patients with oral lichen planus. BMC microbiology. 2020;20(1):1-12.

1124 118. Zhu C, Yuan C, Wei F-Q, Sun X-Y, Zheng S-G. Comparative evaluation of 1125 peptidome and microbiota in different types of saliva samples. Ann Transl Med.

1126 2020;8(11):686-. https://doi.org/10.21037/atm-20-393.

1127 119. Zhu C, Yuan C, Wei FQ, Sun XY, Zheng SG. Intraindividual Variation and Personal 1128 Specificity of Salivary Microbiota. J Dent Res. 2020;99(9):1062-71.

1129 https://doi.org/10.1177/0022034520917155.

1130 120. Ziganshina EE, Sagitov II, Akhmetova RF, Saleeva GT, Kiassov AP, Gogoleva NE, et al. Comparison of the Microbiota and Inorganic Anion Content in the Saliva of Patients with Gastroesophageal Reflux Disease and Gastroesophageal Reflux Disease-Free Individuals. BioMed Research International. 2020;2020:2681791.

1135 https://doi.org/10.1155/2020/2681791. 
A)

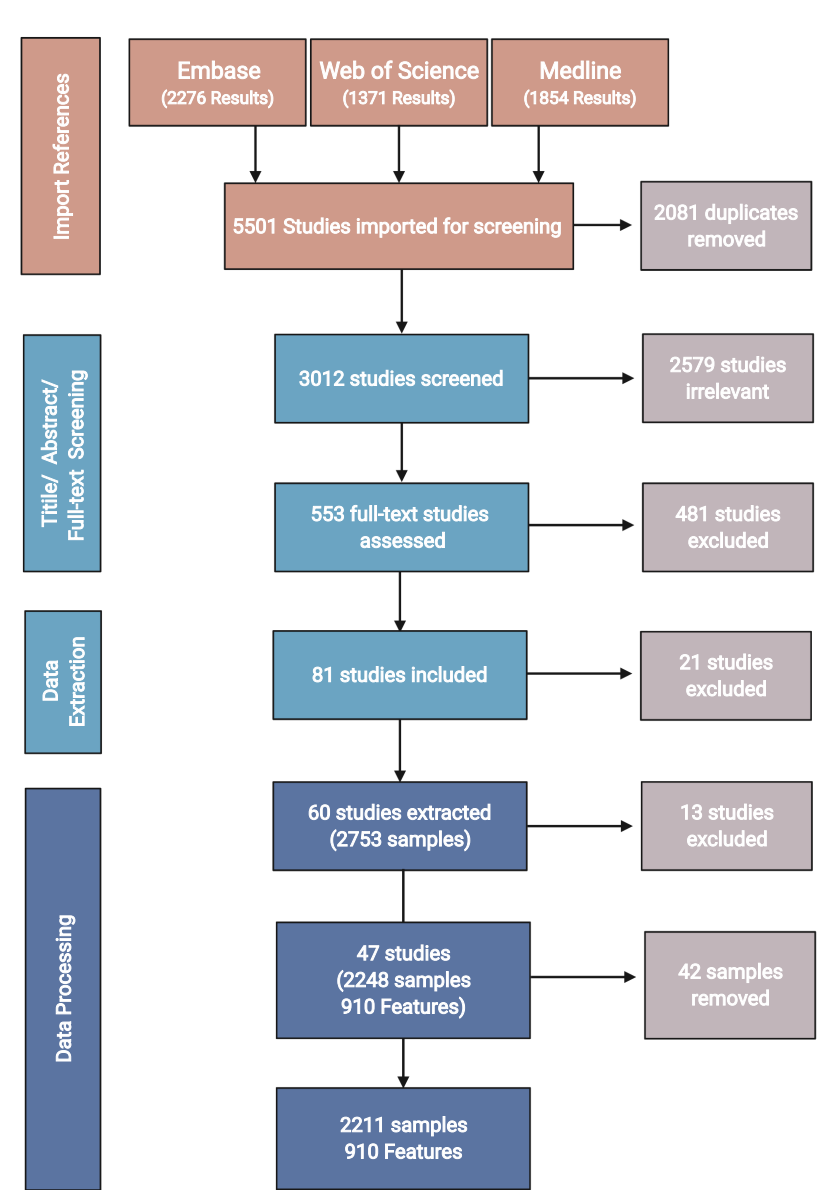

B)

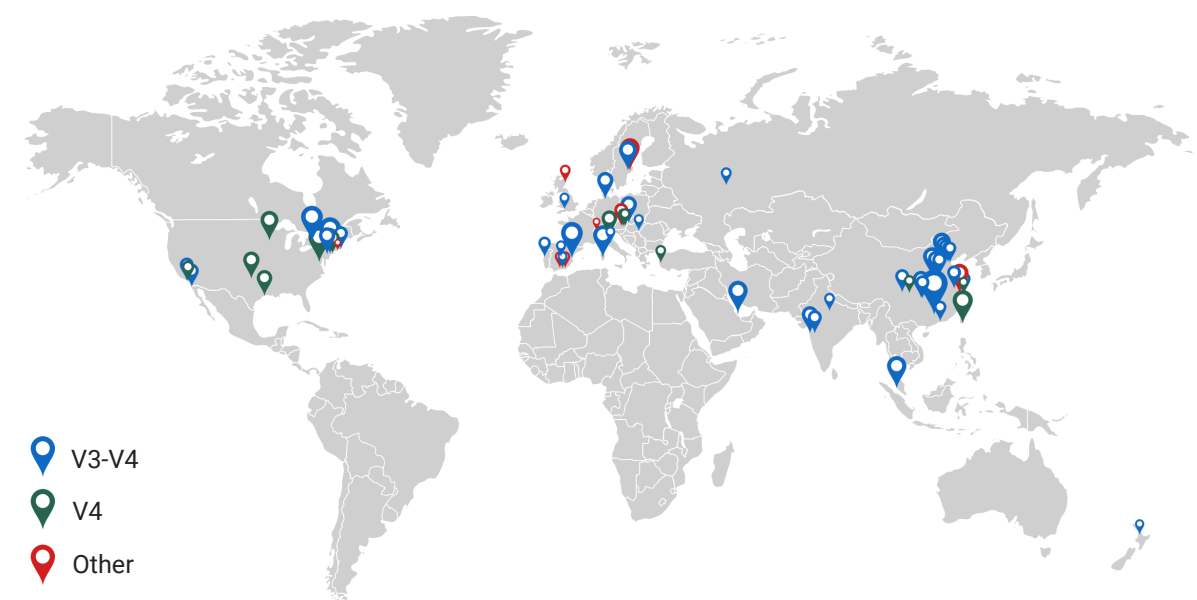

C)

\begin{tabular}{|c|c|c|c|c|}
\hline $\begin{array}{r}\text { Hypervariable } \\
\text { Region }\end{array}$ & 1662 & & & 456 \\
\hline $\begin{array}{r}\text { Geographic } \\
\text { Location }\end{array}$ & 619 & 1012 & & 358 \\
\hline Gender & 653 & 924 & & \\
\hline $\begin{array}{r}\text { Sample } \\
\text { Type }\end{array}$ & 1032 & & 705 & \\
\hline $\begin{array}{r}\text { Age } \\
\text { Range }\end{array}$ & 610 & 547 & 446 & \\
\hline $\begin{array}{c}\text { Tobacco } \\
\text { Usage }\end{array}$ & 519 & & & \\
\hline $\begin{array}{l}\text { Alcohol } \\
\text { Use }\end{array}$ & & & & \\
\hline
\end{tabular}

\begin{tabular}{|c|c|c|}
\hline V3-V4 & V4 & \\
\hline $\begin{array}{c}\text { North } \\
\text { America }\end{array}$ & China & Europe \\
\hline Female & Male & \\
\hline $\begin{array}{c}\text { Un- } \\
\text { stimulated }\end{array}$ & Stimulated & $\begin{array}{c}\text { Mouthwash } \\
\text { (152) }\end{array}$ \\
\hline $18-30$ & $31-50$ & $50+$ \\
\hline $\begin{array}{c}\text { Yes } \\
(139)\end{array}$ & No & \\
\hline $\begin{array}{c}\text { Yes } \\
(149)\end{array}$ & $\begin{array}{c}\text { No } \\
(145)\end{array}$ \\
\hline
\end{tabular}

Figure 1. Overview of literature search procedure and metadata of included studies. a) Large-scale literature searching and data filtering process, followed by the number of samples submitted to the bioinformatic analyses; b) The locations of studies, the scale of symbols that reflect the number of samples of each study; c) Distribution of metadata categories. 
A)

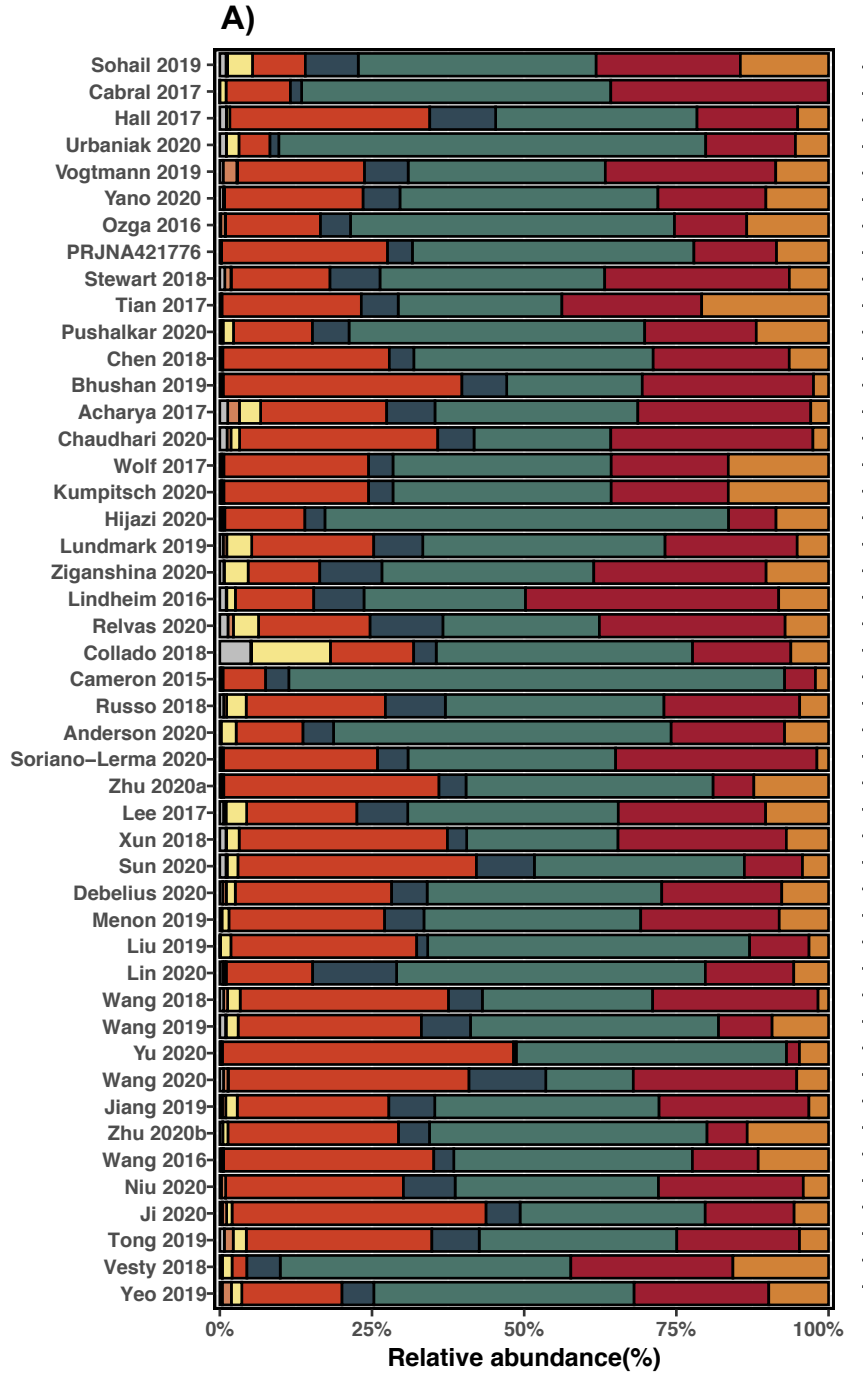

B)

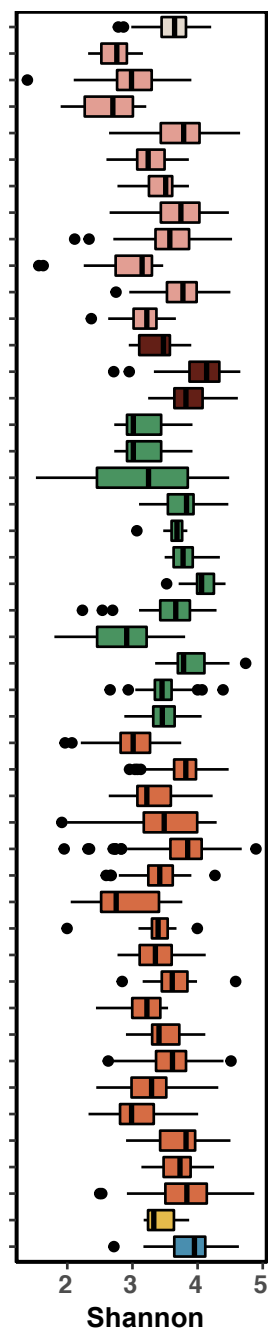

C)

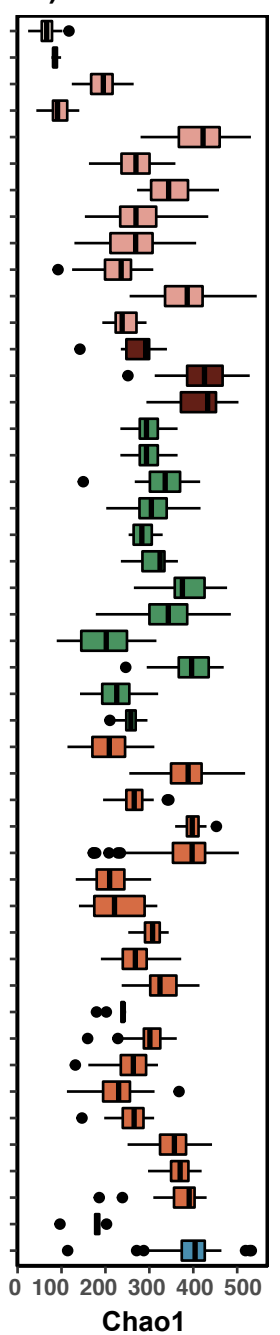

D)

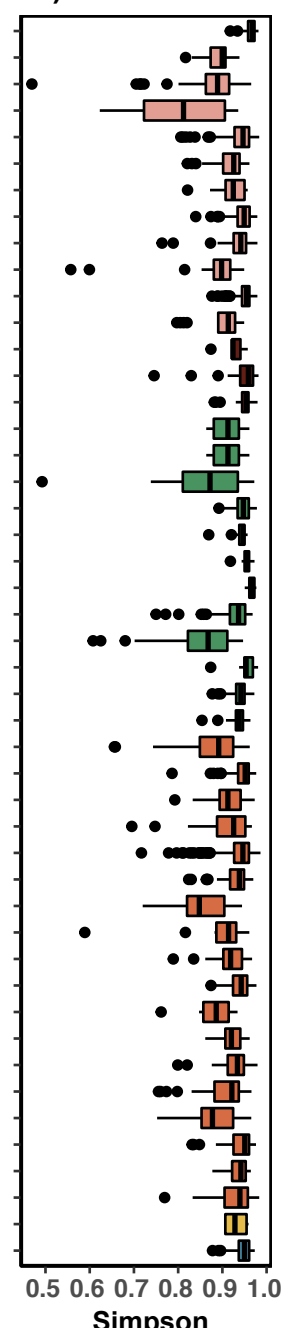

Phylum

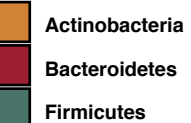

Firmicutes

Fusobacteria

Proteobacteria

Saccharibacteria (TM7)

Spirochaetes

Other

Geographic

Location

身 Malaysia

官 Australia

官 China

官 Europe

India

NorthAmerica

追 Qatar

Figure 2. Summary of taxonomic composition and alpha diversity of included studies. A) The mean community composition of each study at the phylum level; The alpha-diversity measured by B) Shannon index; C) Chao 1 index; D) Simpson's index, the colour of boxes stands for the geographic location of the studies. The horizontal bars within boxes represent medians. The tops and bottoms of boxes represent the 75th and 25th percentiles, respectively. 


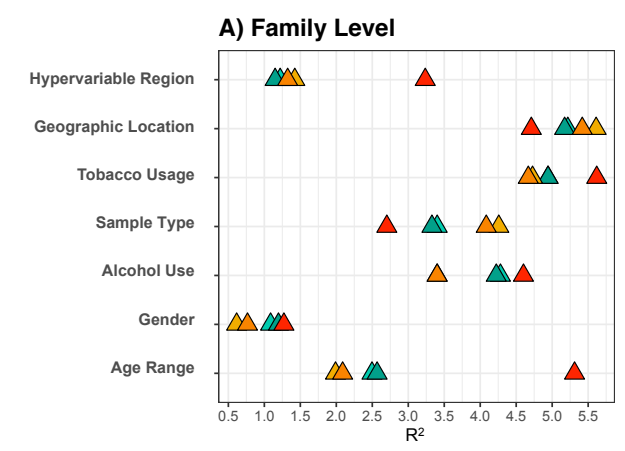

B) Genus Level

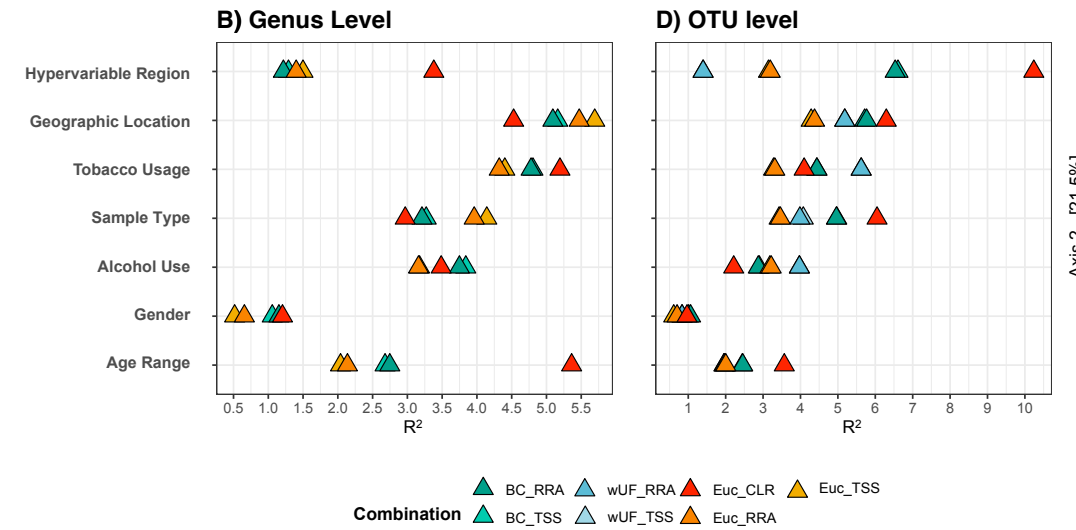

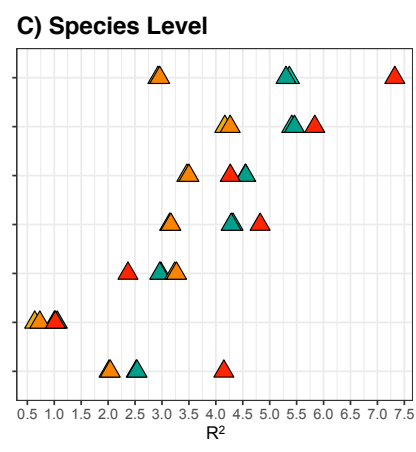

\section{D) OTU level}

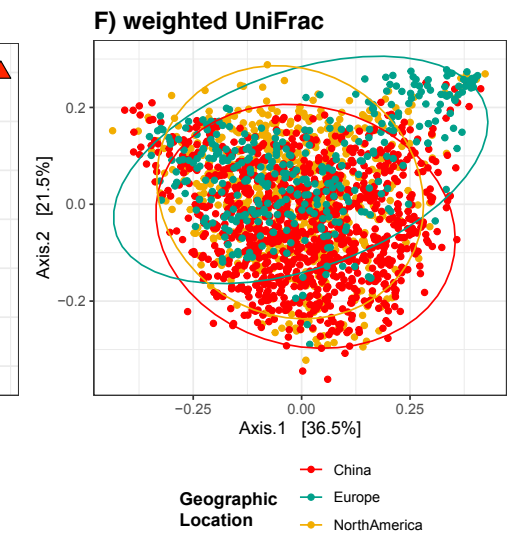

Figure 3. The variability in human salivary microbiota have been explained by different factors. Among them, hypervariable regions and geographic locations have the largest impact. The effect of the categories on the clustering of the sample was measured using PERMANOVA at four taxonomic levels: family (A), Genus (B), species (C) and OTU level (D). The colour indicates the different combinations of normalisation (TSS, Total-sum scaling; RRA, Rarefied relative abundance; CLR, Centred log ratio) and indices (BC, Bray-Curtis; EUC, Euclidean; wUF, weighted uniFrac). Because the results of rarefication (RAR) were very close to TSS and RRA, they were not displayed in the figures. Principal coordinate analysis (PCoA) with Bray-Curtis (E) and weighted uniFrac (F) showing the differences between samples from North America, Europe, and China. 


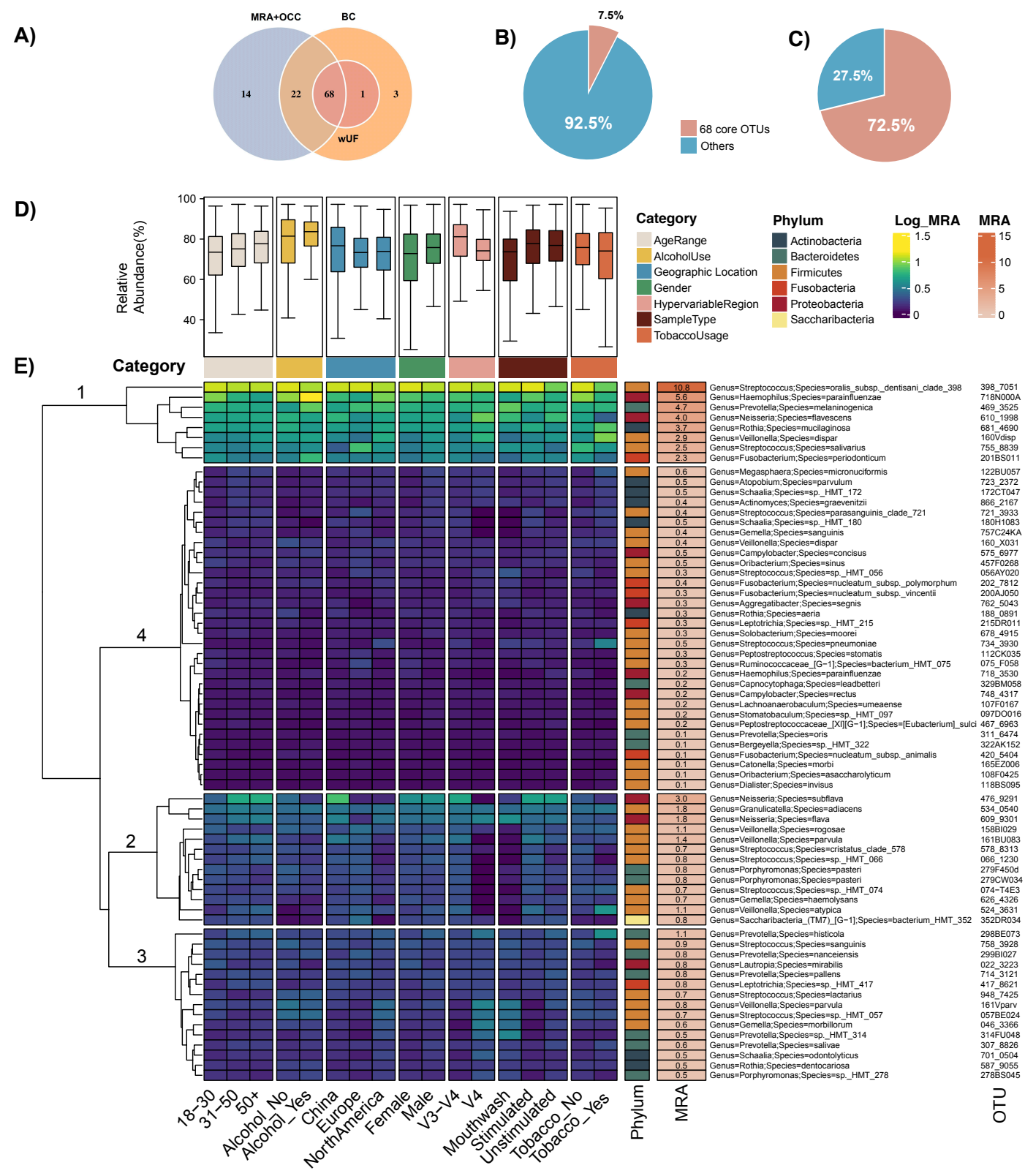

Figure 4. The core OTUs defined by abundance-occupancy pattern. A) Venn diagram showing the interaction between three methods used to define the core. Sixty-eight OTUs were defined as the core for all methods. (MRA+OCC: The thresholds were setting on mean relative abundance and occupancy to define the core; BC: The method adapted from Shade and Stopnisek using Bray-Curtis similarity; wUF: The method adapted from Shade and Stopnisek using weighted uniFrac distance). B) Pie chart showing the number of the core (pink) versus other OTUs (blue) identified in percentage. C) Pie chart showing the relative abundance of the core and other OTUs across all samples. D) Relative abundance of 68 core OTUs across subgroups classified by seven categories. E) Heatmap showing the logtransformed mean relative abundance of each core OTU at each level of different categories. 

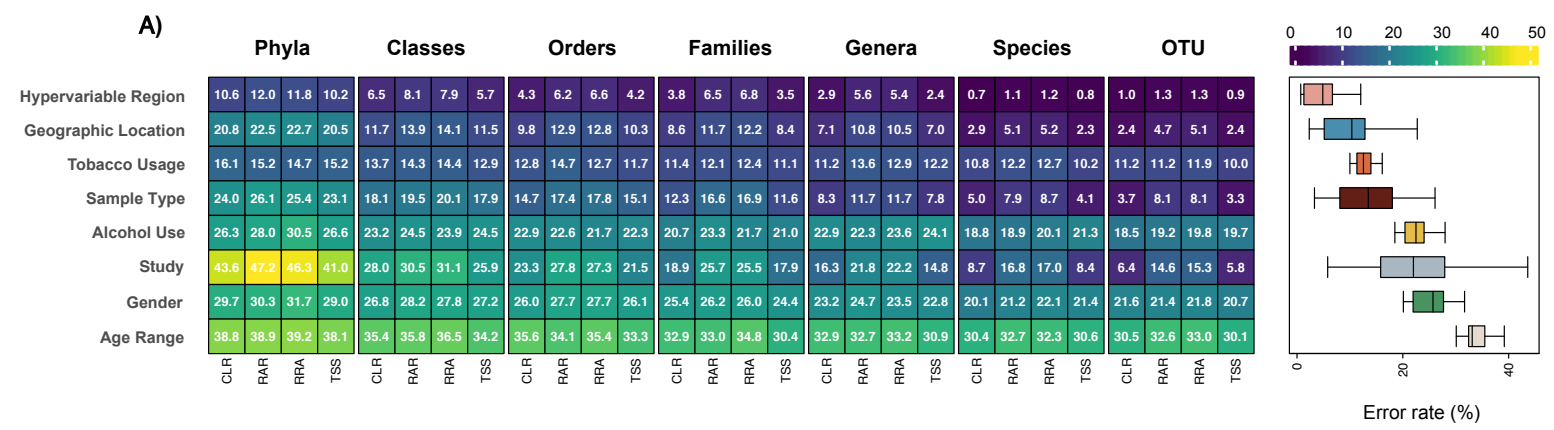

B)
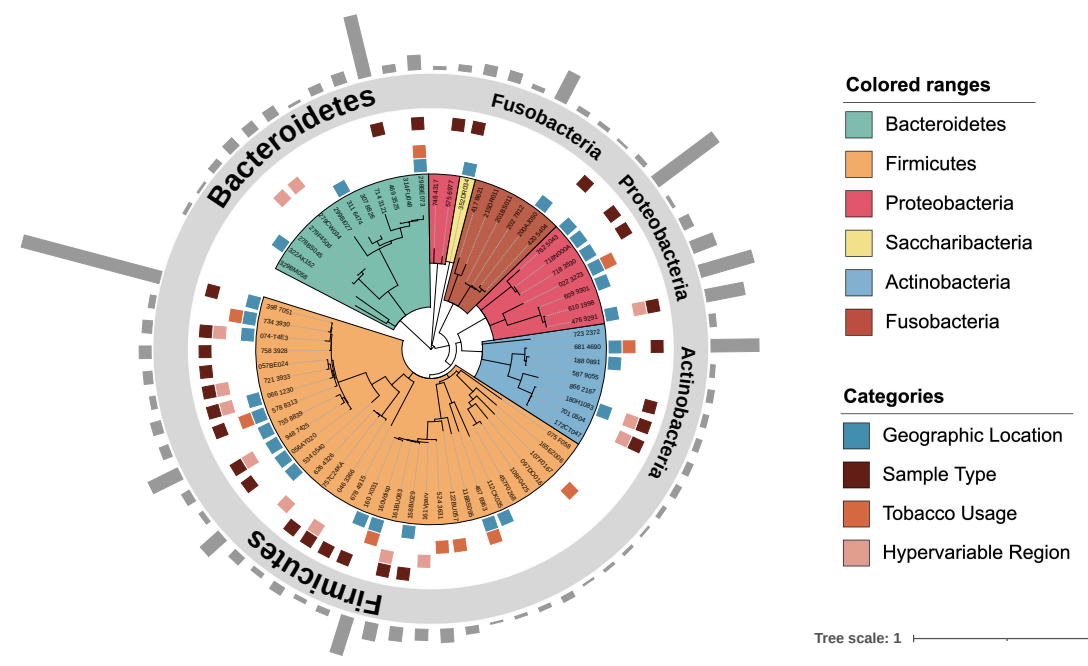

Figure 5. Salivary microbiome members which significantly contribute to categorisation of meta data. Random Forest models showed the impact of categories on salivary microbiome and the core OTUs contributing to accuracy of these models. A) Error rate (\%) for the random forest classifications conducted with samples grouped by eight different categories. B) Phylogenetic tree indicates the taxonomic information of 68 core OTUs. The coloured squares between the tree and the annotation of phylum indicate the OTUs that were defined by the Random Forest model as "important" for distinguishing between different levels in each category. The bars on the outmost ring showing the mean relative abundance of each OTU. 
A)

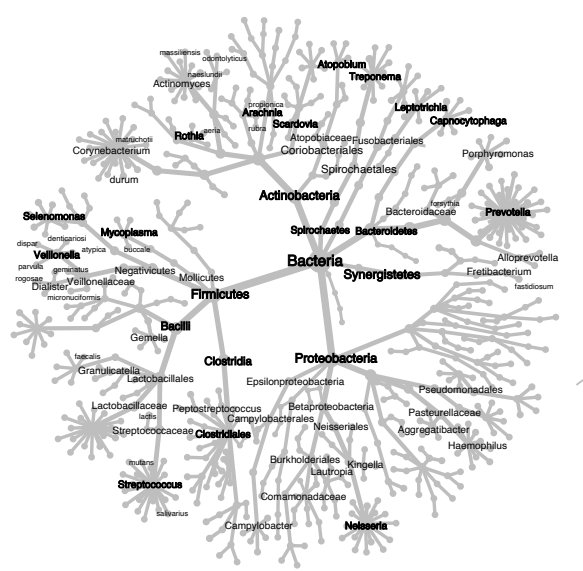

B)

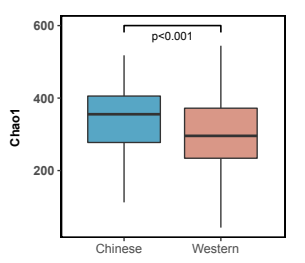

C)

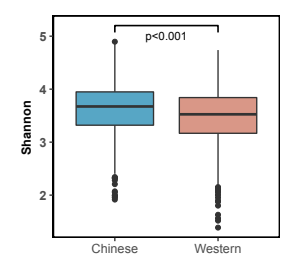

D)
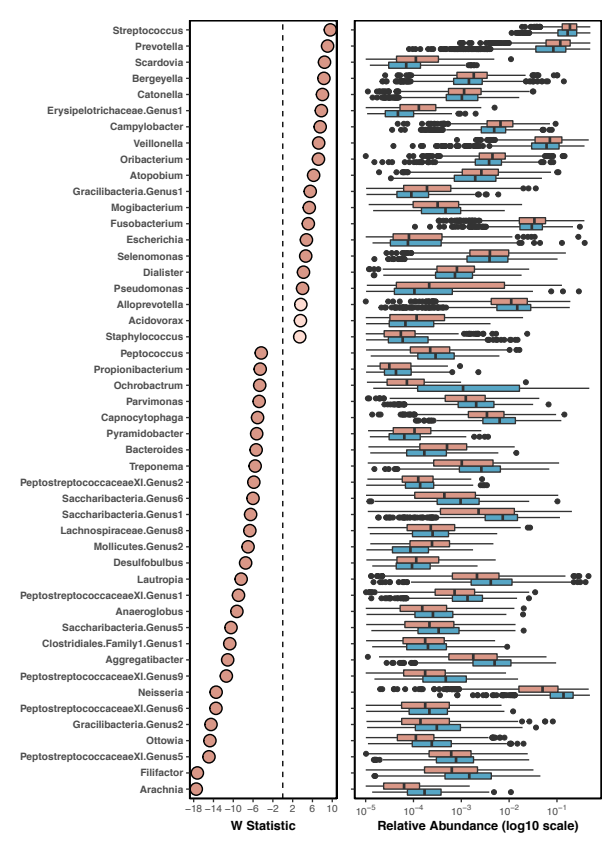

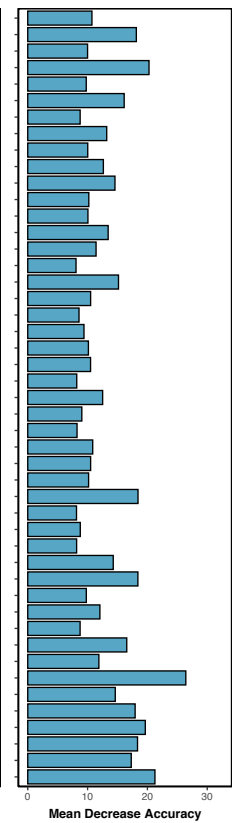

Mean Decrease Accuracy
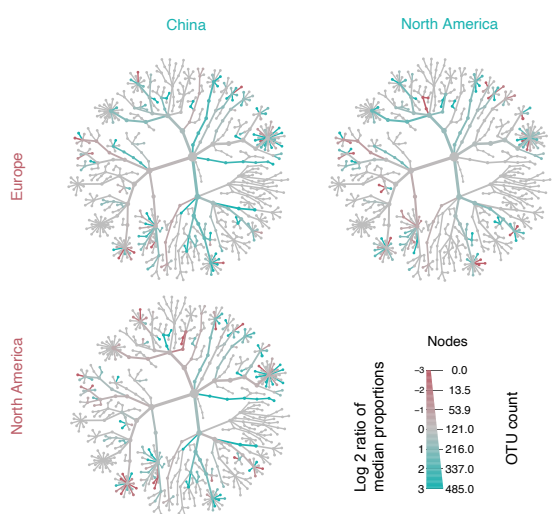

E)
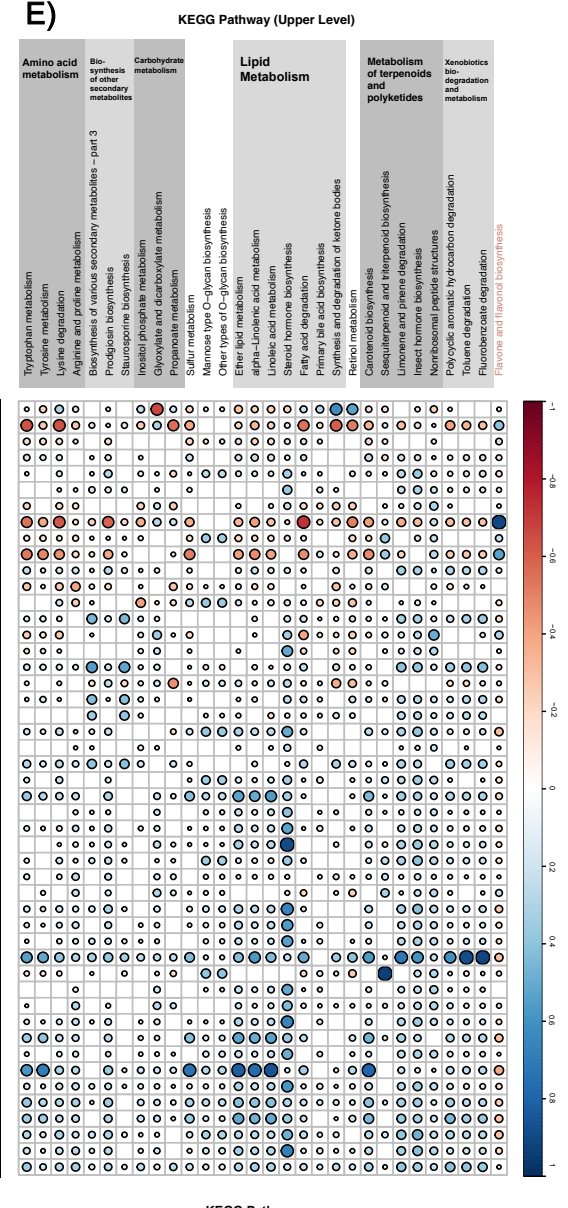

KEGG Pathway

Figure 6. Distinct microbial profiles are evident in the saliva samples from Chinese and Western adults. A) Taxonomic hierarchies show the relative enrichment of taxa in three geographic locations at phylum through species level. Coloured nodes indicate log2-fold increase in median abundance of the group in $\mathrm{x}$-axis (pink) or y-axis (blue). Only taxa showed significant changes (false discovery rate-adjusted Wilcoxon rank sum $\mathrm{q}<0.05$ ) are displayed. B) and C) Comparison of salivary microbial alpha diversity between the Chinese and Western samples, calculated by Chaol (B: $p<0.001$, Wilcoxon rank-sum test) and Shannon index (C: $p<0.001$, Wilcoxon rank-sum test). D) Differential abundant genera identified between saliva from Chinese and Western samples. The panel on the left indicates the standardised effect sizes (W statistic) estimated via the difference on relative abundance using ANCOM-BC (taxa enriched in Western samples have a value shifted to right, whereas taxa enriched in Chinese samples have a value shifted to left); The panel in the middle shows the relative abundance of selected genera; the panel on the right indicates the Mean Decrease Accuracy of the random forest model established. E) Spearman's correlation coefficients were calculated between each pairwise comparison of differential genus and KEGG pathway. Only significantly correlated comparisons ( $\mathrm{p}<0.01$, FDR adjusted Spearman's rank correlation) are displayed. The only Western-enriched pathway is marked in pink. 


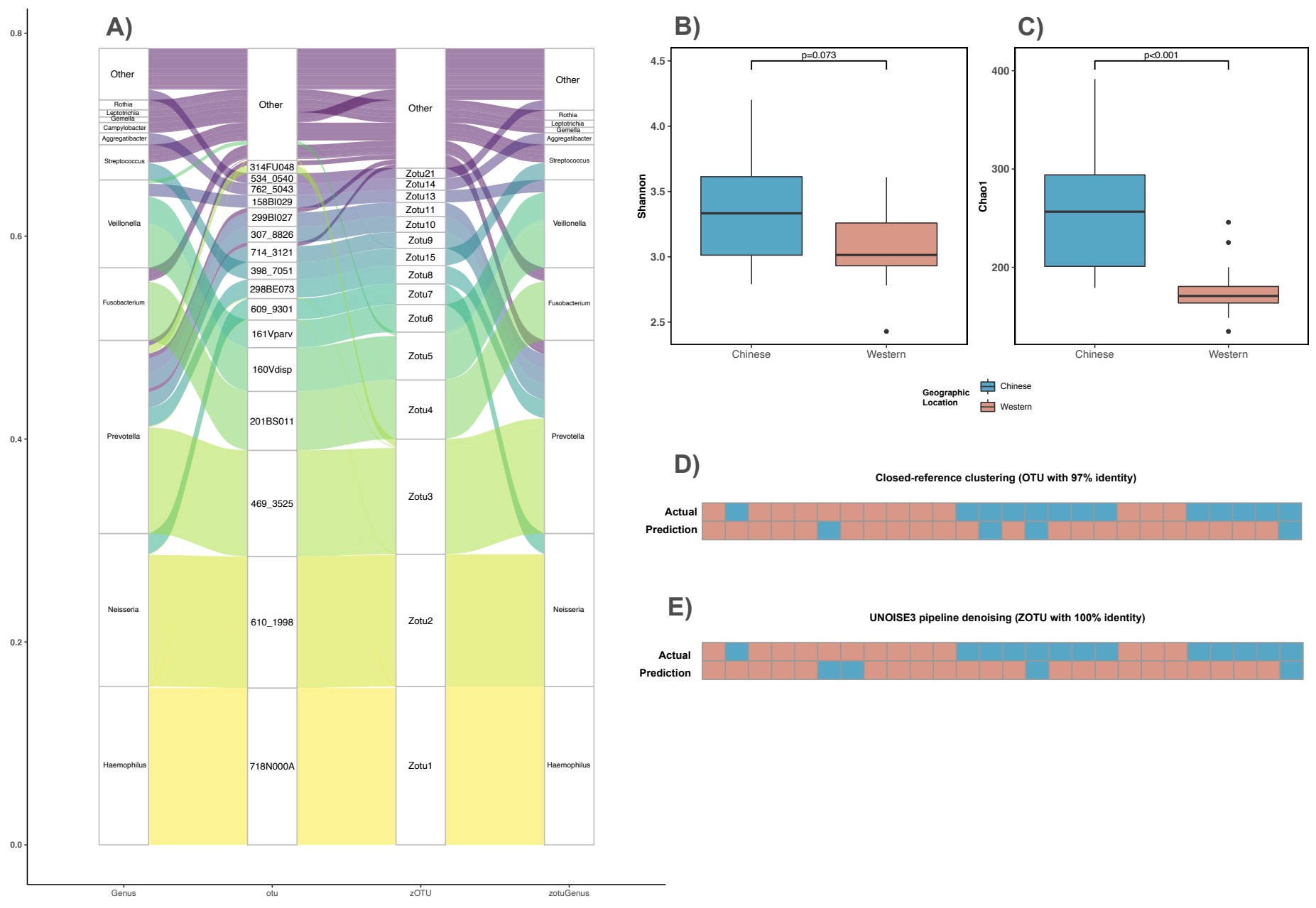

Figure 7. Validation of the findings in the meta-analysis in an independent cohort. A) Alluvial plot showing the affiliation of ZOTUs to their originating core OTUs defined in the meta-analysis. B) and C) Comparison of salivary microbial alpha diversity between the Chinese and Western samples, calculated by Shannon (B: $p=0.073$, Wilcoxon rank-sum test) and Chao1 index (C: $p<0.001$, Wilcoxon rank-sum test). D) and $\mathbf{E})$ The prediction of the cultural backgrounds of the samples according to the random forest classification model constructed using the genus profiles of samples in the meta-analysis. The genus level profiles of samples processed by D) closed-reference clustering with $97 \%$ sequence identity and E) UNOISE3 denoising with $100 \%$ sequence identity were used as the test set. 